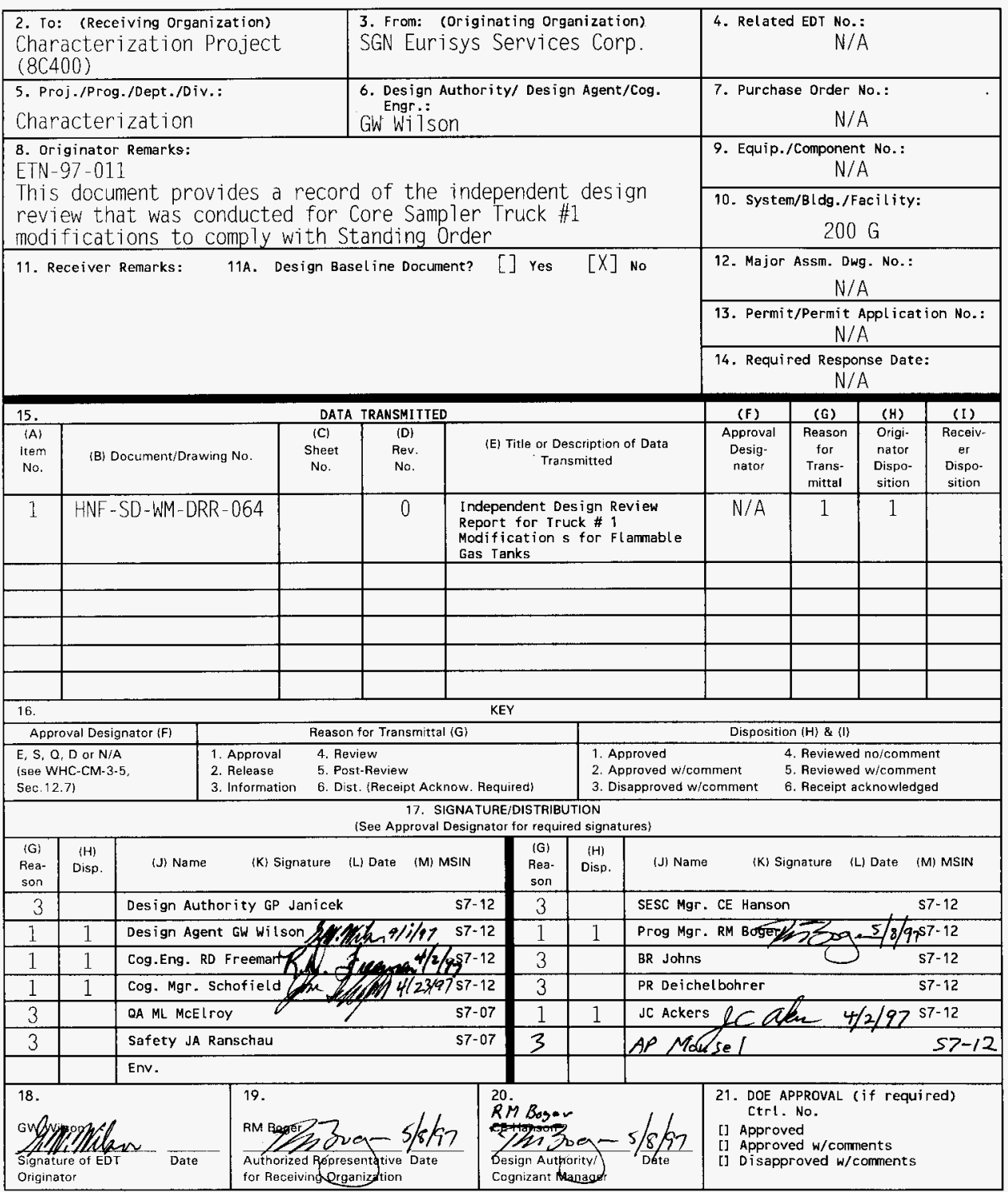

BD-7400-172-2(05/96) GEF097 


\section{INDEPENDENT DESIGN REVIEW REPORT FOR TRUCK \# 1 MODIFICATIONS FOR FLAMMABLE GAS TANKS}

\section{GW Wilson}

SGN Eurisys Services Corp, Richland, WA 99352

U.S. Department of Energy Contract DE-AC06-96RL13200
EDT/ECN: 619143
UC: 2030
Org Code: 08E00
Charge Code: $\mathrm{N} 4 \mathrm{H} 2 \mathrm{~B}$
B\&R Code: EW3120074
Total Pages: $4242 \times \mathrm{kms} 5 \% / 97$

Key Words: CORE, SAMPLE, TRUCK \# 1, FLAMMABLE GAS, RLU, Z PURGE

Abstract: This document records the independent design review that was conducted for Core Sampler Truck \#l modifications to comply with standing order.

TRADEMARK DISCLAIMER. Reference herein to any specific commercial product, process, or service by trade name, trademark, manufacturer, or otherwise, does not necessarjly constitute or imply its endorsement, recommendation, or favoring by the United States Government or any agency thereof or its contractors or subcontractors

Printed in the United States of America. To obtain copies of this document, contact: Document Control Services, P.O. Box 950, Mailstop H6-08, Richland WA 99352, Phone (509) 372-2420; Fax (509) 376-4989.
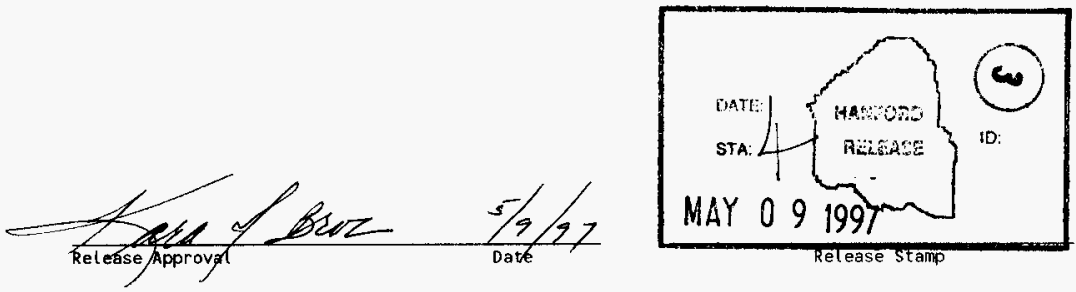

\section{Approved for Public Release}




$$
\begin{aligned}
& \text { HNF-SD-WM-DRR-064, ReV, } 0 \\
& \text { TABLE OF CONTENTS }
\end{aligned}
$$

1.0 sCOPE . . . . . . . . . . . . . . . . . . . . . . . . . 1

2.0 sUMMARY . . . . . . . . . . . . . . . . . . . . . . . . 1

3. 0 DESIGN DESCRIPTION . . . . . . . . . . . . . . . . . . . 2

4.0 LISTING OF REVIEW MATERIALS . . . . . . . . . . . . . . . 3

5. 0 DESIGN COMPLIANCE MATRIX . . . . . . . . . . . . . . . . 3

6.0 REVIEW COMMENT RECORDS . . . . . . . . . . . . . . . . . . 4

7. 0 FGEAB REVIEW . . . . . . . . . . . . . . . . . . . . . . 4

8.0 CONCLUSIONS AND OPEN ITEMS . . . . . . . . . . . . . . . . 5

9. 0 REFERENCES . . . . . . . . . . . . . . . . . . . . . . . 5

10.0 DOCUMENTATION . . . . . . . . . . . . . . . . . . . . . . . 6

ATTACKMENT 1 - Design Compliance Matrix . . . . . . . . . . . 7

ATTACHMENT 2 - Copies of RCR's . . . . . . . . . . . . . . . 13

ATTACHMENT 3 - FGEAB Report . . . . . . . . . . . . . . . . . 35

ATTACKMENT 4 - Final Meeting Minutes . . . . . . . . . . . . 38

ATTACHMENT 5 - Letter from scott . . . . . . . . . . . . . . 40 


$$
\text { HNF-SD-WM-DRR-064， Rev. } 0
$$

\subsection{SCOPE}

This report documents the completion of the independent design review for modifications to the Push Mode core Sample (PMST), Truck \#1, to comply with East and West Tank Farms Standing orders 97-01, Compensatory Actions For open Discovery Unreviewed Safety Questions (USOS). The PMST modifications are to required to support push mode core sampling in waste tanks requiring flammable gas controls. This review includes the Engineering Change Notices (ECNs) for existing drawings which are listed in section 6.0 .

This document and the independent design review are in support of design modifications to PMST used by Characterization project operations (CPO). The modifications are required to allow continued sampling operations in any tanks with flammable gas controls. The objective of this review was to provide a independent design verification consisting of a systematic overall review and evaluation of the modifications. Design verification was performed to insure equipment function, personnel safety, and compliance with WHC-CM-6-1, Standard Engineering practices. Acceptance of the design is required prior to operational use on any waste tanks requiring flammable gas controls.

\subsection{SUMMARY}

The East and West Tank Farm Standing order 97-01 requires that the PMST be modified to include purging of the enclosed space underneath the shielded receiver weather cover per National Fire Protection Association (NFPA) 496, purged and Pressurized Enclosures for Electrical Equipment. The standing order also requires that the PMST also be modified by replacing the existing electrical remote latch (RLU) unit with a mechanical remote latch unit. As the mechanical remote latch unit was exactly like the RLU installed on the Rotary Mode core Sampler Trucks (RMCST) and the design for the RMCST went through formal design review, replacing the RLU was done utilizing informal design verification and was completed per work package ES-97-0028. As the weather cover purge was similar to the design for the RMCSTs, this design was reviewed using the independent review method with multiple independent reviewers. A function design criteria (WHC-SD-WM-FDC-048, Functional Design Criteria for Core Sampling in Flammable Gas Watch List Tanks) provided the criteria for the modifications.

The review consisted of distributing the design review package to the reviewers and collecting and dispositioning the RCR comments. The review package included the ECNs for review, the Design Compliance Matrix, copies of all drawings affected, and copies of outstanding ECNs against these drawings. A final meeting was held 


$$
\text { HNF-SD-WM-DRR-064, Rev. } 0
$$

to ensure that all reviewers were aware of the changes to ECNs from incorporation of RCR comments.

\subsection{DESIGN DEBCRIPTION}

The existing PMST is designed to sample liquid, sludge, and/or salt cake in waste tanks using the universal sampler. During sampling operations, the sampler is retrieved by connecting the shielded receiver to the drill string. The RLU is then used to retrieve the sampler and reinsert an empty sampler. The shielded receiver assembly includes of a sampler hoist box which uses electrical components for power and instrumentation which are located under the shielded receiver weather cover.

To maintain operational and procedural consistency, the decision was made to design the PMST modifications using a type zpressurization system for the weather cover that is similar to what is used on the RMCST.

The type Z-pressurization system which meets requirements of NFPA 496 will consist of a nitrogen purge of approximately 2 SCFM that will be supplied by the nitrogen trailer. The nitrogen will exhaust through two HEPA filters which are located on the bottom of the shielded receiver base plate. The HEPA filters will provide the back pressure to assure compliance with the requirement for a minimum of 0.1 inches water positive pressure in the weather cover. The positive pressure is monitored by two pressure switches which are also located under the shielded receiver base plate. These switches will provide an alarm signal which will activate both alarm lights and an audible alarm at the control console.

The PMST shielded receiver sampler hoist box is different from the RMCSTs in that the sampler hoist box load cell is located outside the sampler hoist box and therefore does not require intrinsic safety barriers for the load cell. The z-pressurization system for the weather cover enclosed space will provide the required protection.

The pre-modification PMST sampler hoist box is vented to the weather cover through a High Efficiency Particulate Air (HEPA) filter. As part of these modifications, this vent was relocated to allow the weather cover enclosed space to be reclassified as a Class 1, Div 2, Group B space and allow the use of a zpressurization system per NFPA 496. The vent is relocated to outside the weather cover with at least 36 leak diameters away from any ignition source.

Also, as part of these modifications, administrative controls will be required in the operating procedures to provide direction for 


$$
\text { HNF-SD-WM-DRR-064， Rev. } 0
$$

equipment start-up, ie. sniffing requirements, and for manual shutdown of equipment after alarm activation.

\subsection{LISTING OF REVIEW MATERIALS}

The following ECNs were reviewed and approved as part of the design review:

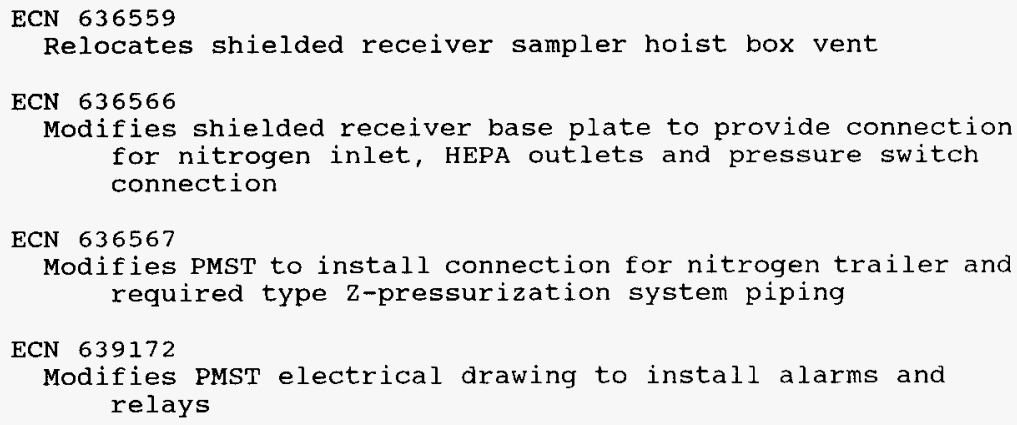

\subsection{DESIGN COMPLIANCE MATRIX}

The design compliance matrix provided as an attachment is section 10.0 was used as a tool for determining that the design met applicable requirements. This design compliance matrix is based on the requirements from the functional design criteria, standing order, and NFPA 496. 


$$
\text { HNF-SD-WM-DRR-064, Rev. } 0
$$

\subsection{REVIEW COMMENT RECORDS}

The following table is a listing of all RCR's received during the independent design review. All RCR's were dispositioned and signed off as closed. Copies of all dispositioned RCRs are included as attachments in section 10.0 of this report.

\begin{tabular}{|l|l|l|}
\hline REVIEWER/DATE/NUMBER & RESP. ENG. & RCR STATUS \\
\hline \hline MCElroy/0421/CP97-008 & GW Wilson & closed/signed \\
\hline McElroy/0421/CP97-009 & GW Wilson & closed/signed \\
\hline MCElroy/0421/CP97-010 & GW Wilson & closed/signed \\
\hline McElroy/0421/CP97-011 & JC Akers & closed/signed \\
\hline Freeman/0424/TF-97-0296 & GW Wilson & closed/signed \\
\hline Freeman/0424/TF-97-0296 & GW Wilson & closed/signed \\
\hline Freeman/0424/TF-97-0296 & GW Wilson & closed/signed \\
\hline Freeman/0424/TF-97-0296 & JC Akers & closed/signed \\
\hline Ranschau/0424/UNNUMBERED & Wilson/Akers & closed/signed \\
\hline Janicek/0421/GPJ-970320 & Wilson/AKers & closed/signed \\
\hline Deichelbohrer/0421/.1 & GW Wilson & closed/signed \\
\hline Johns/0421/1 & JC Akers & closed/signed \\
\hline
\end{tabular}

\subsection{FGEAB REVIEW}

As part of this review, a presentation of these design modification was made to the Flammable Gas Equipment Advisory Board (FGEAB). Their review included reviewing the design for compliance with ignition controls as defined by standing orders. A copy of their report, FGEAB-97-032, is included in section 10.0 


$$
\text { HNF-SD-WM-DRR-064， Rev. } 0
$$

\subsection{CONCLUSIONS AND OPEN ITEMS}

With the approval of this report, the independent review for the PMST modifications for complying with standing orders is completed. The following items were noted during the design review. These items are scheduled to be completed as part of the normal course of business for Acceptance for Beneficial Use (ABU) completion and are not action items for the design review. Therefore these items are NOT considered to be "open items" as described in WHC-CM-6-1, EP 4.1. The Interim classifications of Safety Significant (SS) has been assigned to the Pressure Switches (for $Z$ purge) and Safety Class (SC) to the mechanical Remote Latch Unit (RLU) per WHC-IP0842, TWRS Administration, Volume IV Engineering, section 5.9, Identification of Safety structures, systems, and components, Rev. 0a. The Safety Equipment List (SEL) for the PMST, WHC-SD-WM-SEL023. Safety Classification of Special Equipment for core Sampling Hydrogen Gas and FeCN Watch List Tanks, has been updated (ECN 639152 ) with the new safety designated equipment.

Remaining Items:

- Operating procedure revision required

- New calibration procedure required

- Safety classification criteria needs to be documented

- SEL revision will be required

- ATP/OTP testing required

- $\mathrm{ABU}$ completion/approval required

The conclusion reached by the independent review is that the design of the PMST modifications is acceptable.

\subsection{REFERENCES}

1. WHC-IP-0842, TWRS Administration

2. WHC-SD-WM-6-1, standard Engineering Practices

3. HNF-SD-WM-ETP-221, Engineering Task Plan For Modifying core Sampler Truck \# 1 \& 2 for operation in Flammable Gas Tank

4. WHC-SD-WM-FDC-048, Functional Design Criteria for core Sampling in Flammable Gas Watch List Tanks

5. NFPA 496, Purged and Pressurized Enclosures for Electrical Equipment, 1993

6. East and West Tank Farms standing order 97-01, Compensatory Actions for Open Discovery Unreviewed Safety Questions (USQS), 


$$
\text { HNF-SD-WM-DRR-064, Rev. } 0
$$

(Previously East Tank Farms Standing order 96-36, Compensatory Actions for open Discovery Unreviewed Safety Questions (USQS) \& West Tank Farms standing order 96-34, Compensatory Actions for Open Discovery Unreviewed Safety questions. (USQS))

\subsection{DOCUMENTATION}

The following items are provided as attachments to this report:

1. Design Compliance Matrix

2. Copies of RCR's

3. FGEAB Report

4. Final Review Meeting Minutes

5. Letter/fax from scott (for use of filters with backwards flow, see Ranshau RCR, Item \#2) 
HNF-SD-WM-DRR-064， Rev . 0

ATTACHMENT 1 - Design Compliance Matrix

See the following pages. 


\begin{tabular}{|c|c|c|c|c|c|c|c|c|}
\hline \multicolumn{9}{|c|}{ DESIGN COMPLIANCE MATRIX - TRUCK \# 1 FLAMMABLE GAS MODS } \\
\hline & \multicolumn{4}{|c|}{ REQUIREMENTS } & \multicolumn{2}{|c|}{ SOLUTION } & \multicolumn{2}{|l|}{ VERIFICATION } \\
\hline $\begin{array}{c}\text { Item } \\
\#\end{array}$ & $\begin{array}{c}\text { Class } \\
?\end{array}$ & Criteria & Source & Notes/Assumptions/lmplications & Design Attribute & $\begin{array}{l}\text { Config. } \\
\text { Control } \\
\text { Ref. }\end{array}$ & $\begin{array}{l}\text { Engineering Evaluation } \\
\text { Description }\end{array}$ & $\begin{array}{l}\text { Verified } \\
\text { By? }\end{array}$ \\
\hline $\begin{array}{c}\text { enter } \\
\text { item } \\
\text { no. }\end{array}$ & $\begin{array}{l}\text { enter } \\
\text { SC, } \\
\text { SS, } \\
\text { or, } \\
\text { GS }\end{array}$ & $\begin{array}{l}\text { enter functional or design criteria exactly as } \\
\text { stated in the requirements source document. }\end{array}$ & $\begin{array}{c}\text { enter } \\
\text { source } \\
\text { doc. }\end{array}$ & $\begin{array}{l}\text { enter all additional information which } \\
\text { amplifies, qualifies, justifies,...etc. } \\
\text { the criteria statement. }\end{array}$ & $\begin{array}{l}\text { describe the manner } \\
\text { in which the design } \\
\text { purports to fulfill the } \\
\text { criteria statement - be } \\
\text { as specific as } \\
\text { possible. }\end{array}$ & $\begin{array}{l}\text { provide ref. } \\
\text { for design } \\
\text { attribute } \\
\text { (drawing, } \\
\text { procedure, } \\
\text { spec....etc. } \\
\text { ) }\end{array}$ & $\begin{array}{l}\text { provide discussion on method, } \\
\text { evidence, reasoning used, ...etc., } \\
\text { to verify that the design complies } \\
\text { with requirements - be specific - } \\
\text { include any qualifications, if } \\
\text { appropriate. }\end{array}$ & $\begin{array}{l}\text { person } \\
\text { or group } \\
\text { attesting } \\
\text { to verif. } \\
\text { (initialed) }\end{array}$ \\
\hline 1 & N/A & $\begin{array}{l}\text { Energized electrical equipment that can } \\
\text { come into contact with flammable gases shall } \\
\text { be protected in accordance with NFPA } 496 \\
\text { for use in Class 1, Div } 1 \text {, Group B as defined } \\
\text { by NEC , Article 501. }\end{array}$ & $\begin{array}{c}\text { FDC- } \\
048\end{array}$ & $\begin{array}{l}\text { Equipment in the Drill string, grapple } \\
\text { and sampler hoist box are required } \\
\text { to meet this criteria }\end{array}$ & $\begin{array}{l}\text { Mechanical RLU has } \\
\text { been installed in } \\
\text { Truck \#1. Truck \#1 } \\
\text { has load celt, } \\
\text { switches, and other } \\
\text { electrical equipment } \\
\text { outside of Sampler } \\
\text { Hoist Box under } \\
\text { weather cover. Truck } \\
\text { \#1 Grapple hoist does } \\
\text { not have any internal } \\
\text { electrical equipment. }\end{array}$ & $\begin{array}{l}\mathrm{H}-2-91701 \\
\mathrm{H}-2-91651 \\
\mathrm{H}-2-91390\end{array}$ & $\begin{array}{l}\text { Design meets requirements for } \\
\text { NFPA 496, Type Z } \\
\text { Pressurization as required by } \\
\text { FDC-048 and Standing Order } 97- \\
01 .\end{array}$ & PRD, BRJ \\
\hline 1.2 & $\begin{array}{l}\mathrm{SC}, \\
\text { (INT- } \\
\mathrm{ERIM}\end{array}$ & $\begin{array}{l}\text { The RLU will be modified for use in } \\
\text { flammable gas }\end{array}$ & $\begin{array}{c}\text { FDC- } \\
048 \text { \& } \\
\text { S0 97- } \\
01\end{array}$ & Design Complies & $\begin{array}{l}\text { Mech RLU instailed } \\
\text { per WP ES-97-0028 } \\
\text { in Jan } 1997\end{array}$ & $\begin{array}{l}\mathrm{H}-2-91651 \\
\mathrm{H}-2- \\
690143\end{array}$ & $\begin{array}{l}\text { Design meets requirements as } \\
\text { required by FDC-048 and } \\
\text { Standing Order } 97-01 \text {. }\end{array}$ & PRD \\
\hline 1.3 & N/A & $\begin{array}{l}\text { The Grapple Hoist load cell shall be } \\
\text { protected by using an intrinsic safety barrier }\end{array}$ & $\begin{array}{c}\text { FDC- } \\
048\end{array}$ & $\begin{array}{l}\text { Truck } 1 \text { grapple hoist assy does not } \\
\text { have a load cell therefore } \\
\text { requirement DOES NOT APPLY }\end{array}$ & N/A & $\mathrm{H}-2-91390$ & N/A & N/A \\
\hline 2 & GS & $\begin{array}{l}\text { Electrical equipment that can, under } \\
\text { abnormal conditions, come into contact with } \\
\text { flammable gases shall be protected in } \\
\text { accordance with Standing Order } 97-01 \text { and } \\
\text { NFPA } 496 \text { for use in Class 1, Div 2, Group B } \\
\text { as defined by NEC, Articie } 501 \text {. }\end{array}$ & $\begin{array}{l}\text { FDC- } \\
048\end{array}$ & $\begin{array}{l}\text { The only space affected by this } \\
\text { requirement is underneath the } \\
\text { shielded receiver weather cover } \\
\text { which has numerous and varied } \\
\text { types of electrical equipment. }\end{array}$ & $\begin{array}{l}\text { Sampler hoist box } \\
\text { vent to be moved } \\
\text { outside of weather } \\
\text { cover to reclassify } \\
\text { space. Z-purge } \\
\text { system that complies } \\
\text { to NFPA } 496 \text { to be } \\
\text { installed with } 2 \text { CFM } \\
\text { flow under weather } \\
\text { cover }\end{array}$ & $\begin{array}{l}H-2-91701 \\
H-2-92420 \\
H-2-91663 \\
H-2-92421 \\
H-2-91422\end{array}$ & $\begin{array}{l}\text { Design meets requirements for } \\
\text { NFPA } 496 \text {, Type } Z \\
\text { Pressurization as required by } \\
\text { FDC-048 and Standing Order } 97 \\
01 .\end{array}$ & PRD, BRJ \\
\hline
\end{tabular}


DESIGN COMPLIANCE MATRIX - TRUCK\#1 FLAMMABLE GAS MODS

\begin{tabular}{|c|c|c|c|c|c|c|c|c|}
\hline \multicolumn{9}{|c|}{ DESIGN COMPLIANCE MATRIX - TRUCK\#1 FLAMMABLE GAS MODS } \\
\hline & \multicolumn{4}{|c|}{ REQUIREMENTS } & \multicolumn{2}{|l|}{ SOLUTION } & \multicolumn{2}{|l|}{ VERIFICATION } \\
\hline $\begin{array}{c}\text { Item } \\
\#\end{array}$ & $\begin{array}{c}\text { Class } \\
?\end{array}$ & Criteria & Source & Notes/Assumptions/mplications & Design Attribute & $\begin{array}{l}\text { Config. } \\
\text { Control } \\
\text { Ref. }\end{array}$ & $\begin{array}{c}\text { Engineering Evaluation } \\
\text { Description }\end{array}$ & $\begin{array}{l}\text { Verified } \\
\text { By? }\end{array}$ \\
\hline 2.1 & $\begin{array}{l}\text { SS, } \\
\text { (INT- } \\
\text { ERIM) }\end{array}$ & $\begin{array}{l}\text { The protected enclosure shall be constantly } \\
\text { maintained at a positive pressure of at least } \\
25 \mathrm{~Pa}(0.1 \text { in. water) during operation }\end{array}$ & $\begin{array}{c}\text { NFPA } \\
496 \& \\
\text { SO } 97 \\
01\end{array}$ & $\begin{array}{l}\text { Shielded receiver weather cover } Z \text { - } \\
\text { purge system pressure will be } \\
\text { monitored by two pressure switches } \\
\text { and alarms which will be set to } \\
\text { alarm at less that } 0.5 \text { in water }\end{array}$ & $\begin{array}{l}\text { Two pressure } \\
\text { switches installed on } \\
\text { underside of shielded } \\
\text { receiver base to } \\
\text { monitor pressure and } \\
\text { provide alarm signal. }\end{array}$ & $\mathrm{H}-2-91701$ & $\begin{array}{l}\text { Design meets requirements for } \\
\text { NFPA } 496 \text {, Type } Z \\
\text { Pressurization as required by } \\
\text { FDC-048 and Standing Order } 97 \\
01 .\end{array}$ & PRD, BRJ \\
\hline 2.2 & GS & $\begin{array}{l}\text { An alarm shall be provide to indicate failure } \\
\text { of protective gas supply to maintain required } \\
\text { pressure }\end{array}$ & $\begin{array}{c}\text { NFPA } \\
4968 \\
\text { SO } 97 \\
01\end{array}$ & $\begin{array}{l}\text { Shielded receiver weather cover Z- } \\
\text { purge system will supply } \\
\text { approximately } 2 \text { SCFM nitrogen to } \\
\text { weather cover enclosed space and } \\
\text { pressure will be monitored by two } \\
\text { pressure switches connected to } \\
\text { alarms }\end{array}$ & $\begin{array}{l}\text { An alarm light and } \\
\text { audible alarm ( } 80 \\
\text { Db) with } \\
\text { acknowledge button } \\
\text { will be installed in } \\
\text { control console. }\end{array}$ & $\begin{array}{l}\mathrm{H}-2-91420 \\
\mathrm{H}-2-91421 \\
\mathrm{H}-2-91422\end{array}$ & $\begin{array}{l}\text { Design meets requirements for } \\
\text { NFPA } 496 \text {, Type } Z \\
\text { Pressurization as required by } \\
\text { FDC-048 and Standing Order } 97- \\
01 .\end{array}$ & PRD, BRJ \\
\hline 2.2 .1 & $\overline{G S}$ & $\begin{array}{l}\text { The alarm and/or indicator shall meet } \\
\text { requirements of NFPA } 496 \text { for type } Z \\
\text { Pressurization }\end{array}$ & $\begin{array}{c}\text { NFPA } \\
496 \& \\
\text { SO } 97 \\
01\end{array}$ & $\begin{array}{l}\text { Alarm will only need to be attended } \\
\text { when shielded receiver is connected } \\
\text { to drill string }\end{array}$ & $\begin{array}{l}\text { The alarm and } \\
\text { indicator will be install } \\
\text { in the control console } \\
\text { which is where the } \\
\text { operator is during } \\
\text { sample retrieval. The } \\
\text { alarm signal comes } \\
\text { from pressure } \\
\text { switches which } \\
\text { monitor positive } \\
\text { pressure under the } \\
\text { shielded receiver } \\
\text { weather cover. }\end{array}$ & $\begin{array}{l}H-2-91421 \\
H-2-91422\end{array}$ & $\begin{array}{l}\text { Design meets requirements for } \\
\text { NFPA } 496 \text {, Type } Z \\
\text { Pressurization as required by } \\
\text { FDC-048 and Standing Order } 97 \text { - } \\
01 .\end{array}$ & BRJ \\
\hline 2.2 .2 & GS & $\begin{array}{l}\text { All controls and displays shall be located } \\
\text { where they can be clearly seen and easily } \\
\text { reached. all lights shall be visible in bright or } \\
\text { hazy sunlight. }\end{array}$ & $\begin{array}{c}\text { FDG- } \\
048\end{array}$ & $\begin{array}{l}\text { Control console is used for normal } \\
\text { operation of sampler hoist and is } \\
\text { easily accessible. Lights for } \\
\text { shielded receiver weather cover Z } \\
\text { Pressurization will be one green } \\
\text { light for pressurization OK and one } \\
\text { red light to indicate pressurization } \\
\text { failure. }\end{array}$ & $\begin{array}{l}\text { Lights and controls } \\
\text { will be located in } \\
\text { control console and } \\
\text { will be equivalent to } \\
\text { existing lights. }\end{array}$ & H-2-91421 & $\begin{array}{l}\text { Design meets requirements for } \\
\text { NFPA } 496 \text {, Type } Z \\
\text { Pressurization as required by } \\
\text { FDC-048 and Standing Order } 97 \text { - } \\
01 .\end{array}$ & BRJ \\
\hline
\end{tabular}


DESIGN COMPLIANCE MATRIX - TRUCK \#1 FLAMMABLE GAS MODS

\begin{tabular}{|c|c|c|c|c|c|c|c|c|}
\hline \multicolumn{9}{|c|}{ DESIGN COMPLIANCE MATRIX - TRUCK\# 1 FLAMMABLE GAS MODS } \\
\hline & \multicolumn{4}{|c|}{ REQUIREMENTS } & \multicolumn{2}{|l|}{ SOLUTION } & \multicolumn{2}{|l|}{ VERIFICATION } \\
\hline $\begin{array}{c}\text { Item } \\
\#\end{array}$ & $\begin{array}{c}\text { Class } \\
?\end{array}$ & Criteria & Source & Notes/Assumptions/lmplications & Design Altribute & $\begin{array}{l}\text { Config. } \\
\text { Control } \\
\text { Ref. }\end{array}$ & $\begin{array}{c}\text { Engineering Evaluation } \\
\text { Description }\end{array}$ & $\begin{array}{l}\text { Verified } \\
\text { By? }\end{array}$ \\
\hline 2.3 & GS & $\begin{array}{l}\text { Instrument quality air, nitrogen, or other } \\
\text { nonflammable gas shall be considered } \\
\text { acceptable as a protective gas }\end{array}$ & \begin{tabular}{c|} 
NFPA \\
496 \& \\
SO $97-$ \\
01
\end{tabular} & Design complies & $\begin{array}{l}\text { Truck \# } 1 \text { to be } \\
\text { modified with addition } \\
\text { of connection for the } \\
\text { existing nitrogen } \\
\text { purge trailers }\end{array}$ & H-2-91701 & $\begin{array}{l}\text { Design meets requirements for } \\
\text { NFPA 496, Type Z } \\
\text { Pressurization as required by } \\
\text { FDC-048 and Standing Order } 97- \\
01 .\end{array}$ & PRD, BRJ \\
\hline 2.4 & GS & $\begin{array}{l}\text { A permanent label shall be mounted on the } \\
\text { protected enclosure in prominent location to } \\
\text { say: WARNING - PRESSURIZED } \\
\text { ENCLOSURE }\end{array}$ & $\begin{array}{c}\text { NFPA } \\
496 \text { \& } \\
\text { SO } 97- \\
01\end{array}$ & Design Complies & $\begin{array}{l}\text { Truck \# } 1 \text { shielded } \\
\text { receiver weather } \\
\text { cover will be labeled } \\
\text { per requirements }\end{array}$ & $\mathrm{H}-2-91651$ & $\begin{array}{l}\text { Design meets requirements for } \\
\text { NFPA } 496 \text {, Type } Z \\
\text { Pressurization as required by } \\
\text { FDC-048 and Standing Order } 97- \\
01 .\end{array}$ & PRD \\
\hline 3 & N/A & $\begin{array}{l}\text { Motors shall not be energized until at least } \\
10 \text { volumes have passed through enclosure } \\
\text { while maintaining internal pressure. }\end{array}$ & \begin{tabular}{|c|} 
NFPA \\
$496 \&$ \\
SO 97 \\
01
\end{tabular} & $\begin{array}{l}\text { EXCEPTION: Equipment shall be } \\
\text { permitted to be energized } \\
\text { immediately if required pressure } \\
\text { exists and the atmosphere within the } \\
\text { enclosure is known to be } \\
\text { nonflammable (Quote from NFPA } \\
496 \text { ) }\end{array}$ & $\begin{array}{l}\text { Normal operation } \\
\text { only provides } \\
\text { potential for } \\
\text { flammable gas during } \\
\text { sampler retrieval } \\
\text { operations. Normal } \\
\text { condition during all } \\
\text { other operations } \\
\text { results in known non } \\
\text { flammable gas } \\
\text { atmosphere under } \\
\text { shielded receiver } \\
\text { weather cover }\end{array}$ & TO-080-503 & $\begin{array}{l}\text { The procedure is being revised } \\
\text { to require "sniffing" of enclosed } \\
\text { space before initial start-up. }\end{array}$ & $\mathrm{RDF}$ \\
\hline 4 & $\mathrm{~N} / \mathrm{A}$ & $\begin{array}{l}\text { All ignition sources not normally in contact } \\
\text { with waste or waste gases shall be placed at } \\
\text { least } 36 \text { leak diameters from open leak } \\
\text { sources }\end{array}$ & $\begin{array}{l}\text { FDC- } \\
048\end{array}$ & $\begin{array}{l}\text { Requires that sampler hoist vent be } \\
\text { located away from engine exhaust } \\
\text { pipe }\end{array}$ & $\begin{array}{l}\text { Sampler hoist vent to } \\
\text { be located } 36 \text { inches } \\
\text { from engine exhaust } \\
\text { pipe }\end{array}$ & $\mathrm{H}-2-91651$ & $\begin{array}{l}\text { Design meets requirements in } \\
\text { FDC-048. }\end{array}$ & $\overline{P R D}$ \\
\hline 5 & N/A & $\begin{array}{l}\text { Acceptable safe shutdown of equipment not } \\
\text { located in Class } 1 \text {, Div } 1 \text { may be by manual } \\
\text { jactions }\end{array}$ & $\begin{array}{c}\text { FDC- } \\
\text { O48, } \\
\text { NFPA } \\
496 \text { \& } \\
\text { SO } 97 \\
01\end{array}$ & Design Complies & $\begin{array}{l}\text { Manual shutdown of } \\
\text { sampler hoist } \\
\text { electrical equipment } \\
\text { will be addressed in } \\
\text { operating procedure } \\
\text { and will be verified at } \\
\text { ABU signoff }\end{array}$ & $\begin{array}{l}\mathrm{H}-2-91421 \\
\mathrm{H}-2-91422 \\
\mathrm{H}-2-91420 \\
\mathrm{TO}-080-503\end{array}$ & $\begin{array}{l}\text { Design meets requirements for } \\
\text { NFPA } 496, \text { Type } Z \\
\text { Pressurization as required by } \\
\text { FDC-048 and Standing Order } 97- \\
01 .\end{array}$ & BRJ \\
\hline 6 & $\mathrm{~N} / \mathrm{A}$ & $\begin{array}{l}\text { Safe shutdown configuration shall prevent } \\
\text { hydrogen accumulation in the piping system. } \\
\text { All purge gas shall be routed back to the } \\
\text { waste tank, or through approved filtration }\end{array}$ & $\begin{array}{c}\text { FDC- } \\
048\end{array}$ & $\begin{array}{l}\text { During normal shutdown shielded } \\
\text { receiver tube is disconnected from } \\
\text { drill string therefore is not classified } \\
\text { space. }\end{array}$ & $\begin{array}{l}\text { Z-purge gases are } \\
\text { exhausted through } \\
\text { two assault mask } \\
\text { HEPA filters }\end{array}$ & H-2-91701 & $\begin{array}{l}\text { Design meets requirements in } \\
\text { FDC-048. }\end{array}$ & PRD \\
\hline
\end{tabular}


DESIGN COMPLIANCE MATRIX - TRUCK \# 1 FLAMMABLE GAS MODS

\begin{tabular}{|c|c|c|c|c|c|c|c|c|}
\hline \multicolumn{9}{|c|}{ DESIGN COMPLIANCE MATRIX - TRUCK \# 1 FLAMMABLLE GAS MODS } \\
\hline & \multicolumn{4}{|c|}{ REQUIREMENTS } & \multicolumn{2}{|l|}{ SOLUTION } & \multicolumn{2}{|l|}{ VERIFICATION } \\
\hline $\begin{array}{c}\text { Item } \\
\#\end{array}$ & Class & Criteria & Source & Notes/Assumptions//mplications & Design Attribute & $\begin{array}{l}\text { Config. } \\
\text { Control } \\
\text { Ref. }\end{array}$ & $\begin{array}{l}\text { Engineering Evaluation } \\
\text { Description }\end{array}$ & $\begin{array}{l}\text { Verified } \\
\text { By? }\end{array}$ \\
\hline 7 & $\bar{N} / A$ & $\begin{array}{l}\text { The shielded receiver weather cover shall be } \\
\text { modified to prevent hydrogen accumulation. } \\
\text { Options include cover removal, venting } \\
\text { provisions, and/or Z-purge }\end{array}$ & $\begin{array}{c}\text { FDC- } \\
\text { O48, } \\
\text { NFPA } \\
496\end{array}$ & Design Complies & $\begin{array}{l}\text { Z-purge system being } \\
\text { installed for shielded } \\
\text { receiver weather } \\
\text { cover that meets } \\
\text { requirements of } \\
\text { NFPA } 496 \text {. Sampler } \\
\text { hoist vent is } \\
\text { relocation to remove } \\
\text { potential flammable } \\
\text { gases from the } \\
\text { shielded receiver } \\
\text { weather cover }\end{array}$ & $\mathrm{H}-2-91701$ & $\begin{array}{l}\text { Design meets requirements for } \\
\text { NFPA } 496 \text {, Type } Z \\
\text { Pressurization as required by } \\
\text { FDC-048 and Standing Order } 97 \text {. } \\
01 .\end{array}$ & PRD, BRJ \\
\hline 8 & $\tilde{N} / A$ & $\begin{array}{l}\text { The load cell instrument associated with the } \\
\text { shieided receiver shall be protected using } \\
\text { intrinsic safety barriers }\end{array}$ & $\begin{array}{c}\text { FDC- } \\
048\end{array}$ & $\begin{array}{l}\text { Truck } 1 \text { sampler hoist load cell is } \\
\text { located outside sampler hoist box } \\
\text { under shielded receiver weather } \\
\text { cover which is a Class } 1 \text {, Div } 2 \\
\text { space }\end{array}$ & $\begin{array}{l}\text { Z-purge system being } \\
\text { installed for shielded } \\
\text { receiver weather } \\
\text { cover }\end{array}$ & $\mathrm{H}-2-91651$ & $\begin{array}{l}\text { Design meets requirements in } \\
\text { FDC- } 048 \text {. }\end{array}$ & PRD, BRJ \\
\hline 9 & N/A & $\begin{array}{l}\text { The acceptability of design to include } \\
\text { features of performance alternates (ie.. } \\
\text { redundancy, by-pass administrative controls) } \\
\text { shall be evaluated by considering single } \\
\text { active failures of components. }\end{array}$ & $\begin{array}{c}\text { FDC- } \\
048\end{array}$ & $\begin{array}{l}\text { Positive pressure is provided by Z- } \\
\text { purge system which supplies } \\
\text { approximately } 2 \text { SCFM nitrogen to } \\
\text { shielded receiver weather cover and } \\
\text { is exhausted through two mask } \\
\text { HEPA filters which will provide } \\
\text { required back pressure }\end{array}$ & $\begin{array}{l}\text { Redundant pressure } \\
\text { switches and alarms } \\
\text { are used to ensure } \\
\text { that positive pressure } \\
\text { is maintained under } \\
\text { shielded receiver } \\
\text { weather cover }\end{array}$ & H-2-91701 & $\begin{array}{l}\text { Design meets requirements in } \\
\text { FDC- } 048 \text {. }\end{array}$ & PRD \\
\hline 10 & N/A & $\begin{array}{l}\text { The CSS shall be designed to provide for } \\
\text { confinement barriers and air filtration } \\
\text { systems for contarnination control }\end{array}$ & $\begin{array}{l}\text { FDC- } \\
048\end{array}$ & Design Complies & $\begin{array}{l}\text { Z-purge gas is } \\
\text { exhausted through } \\
\text { two assault mask } \\
\text { HEPA filters }\end{array}$ & $\mathrm{H}-2-91663$ & $\begin{array}{l}\text { Design meets requirements in } \\
\text { FDC-048. }\end{array}$ & PRD \\
\hline 11 & N/A & $\begin{array}{l}\text { The system shall ensure that a single failure } \\
\text { does not result in the loss of capability of a } \\
\text { safety class system to perform its safety } \\
\text { function. }\end{array}$ & $\begin{array}{l}\text { FDC- } \\
048\end{array}$ & $\begin{array}{l}\text { Pressure switch failure, cable failure, } \\
\text { relay failure or power failure to } \\
\text { pressure switches will cause alarms }\end{array}$ & $\begin{array}{l}\text { Redundant pressure } \\
\text { switches and alarms } \\
\text { are provided to } \\
\text { ensure that the Z- } \\
\text { purge system } \\
\text { functions }\end{array}$ & $\mathrm{H}-2-91701$ & $\begin{array}{l}\text { Design meets requirements in } \\
\text { FDC-048. }\end{array}$ & PRD \\
\hline 12 & $\mathrm{~N} / \mathrm{A}$ & $\begin{array}{l}\text { Al aluminum potentially in contact with tank } \\
\text { waste shall be eliminated. }\end{array}$ & $\begin{array}{c}\text { FDC- } \\
048\end{array}$ & $\begin{array}{l}\text { No aluminum parts inside sampler } \\
\text { hoist box, grapple box, or RLU }\end{array}$ & No aluminum parts & $\begin{array}{l}\mathrm{H}-2-91651 \\
\mathrm{H}-2-690143 \\
\mathrm{H}-2-91390\end{array}$ & $\begin{array}{l}\text { Design meets requirements in } \\
\text { FDC-048. }\end{array}$ & PRD \\
\hline
\end{tabular}




\begin{tabular}{|c|c|c|c|c|c|c|c|c|}
\hline \multicolumn{9}{|c|}{ DESIGN COMPLIANCE MÄTRIX - TRUCK \# 1 FLAMMABLE GAS MÓDS } \\
\hline & \multicolumn{4}{|c|}{ REQUIREMENTS } & \multicolumn{2}{|c|}{ SOLUTION } & \multicolumn{2}{|l|}{ VERIFICATION } \\
\hline $\begin{array}{c}\text { Item } \\
\#\end{array}$ & $\begin{array}{c}\text { Class } \\
?\end{array}$ & Criteria & Source & Notes/Assumptions/mplications & Design Attribute & $\begin{array}{l}\text { Config. } \\
\text { Control } \\
\text { Ref. }\end{array}$ & $\begin{array}{l}\text { Engineering Evaluation } \\
\text { Description }\end{array}$ & $\begin{array}{l}\text { Verified } \\
\text { By? }\end{array}$ \\
\hline 13 & GS & $\begin{array}{l}\text { Operating temperature range from } 10 \text { to } 100 \\
\text { degrees } F \text {. Non operating temperature range } \\
\text { from }-20 \text { to } 115 \text { degrees } F \text {. }\end{array}$ & $\begin{array}{c}\text { FOC- } \\
048\end{array}$ & $\begin{array}{l}\text { Switches, lights, alarms etc. being } \\
\text { added are equivalent to existing } \\
\text { equipment on Core Sampling Trucks }\end{array}$ & $\begin{array}{l}\text { Control console is } \\
\text { heated and cooled } \\
\text { during operations. }\end{array}$ & $\begin{array}{l}\mathrm{H}-2-92420 \\
\mathrm{H}-2-91663\end{array}$ & $\begin{array}{l}\text { Design meets requirements in } \\
\text { FDC-048. }\end{array}$ & BRJ \\
\hline 14 & GS & $\begin{array}{l}\text { Operating range in winds of up to } 15 \mathrm{MPH} \text { in } \\
\text { blowing dust, sand, rain, and snow. }\end{array}$ & $\begin{array}{c}\text { FDC- } \\
048\end{array}$ & $\begin{array}{l}\text { Wind could cause bursting of burst } \\
\text { disc. Other new equipment is } \\
\text { equivalent to existing equipment. }\end{array}$ & $\begin{array}{l}\text { Burst disc is located } \\
\text { in shielded location } \\
\text { (under base plate) } \\
\text { and outlet is cover } \\
\text { with sheet metal } \\
\text { baffle. }\end{array}$ & $\mathrm{H}-2-91663$ & $\begin{array}{l}\text { Design meets requirements in } \\
\text { FDC-048. }\end{array}$ & PRD \\
\hline
\end{tabular}


HNF-SD-WM-DRR-064， Rev. 0

ATTACHMENT 2 - Copies of RCR's

see the following pages. 


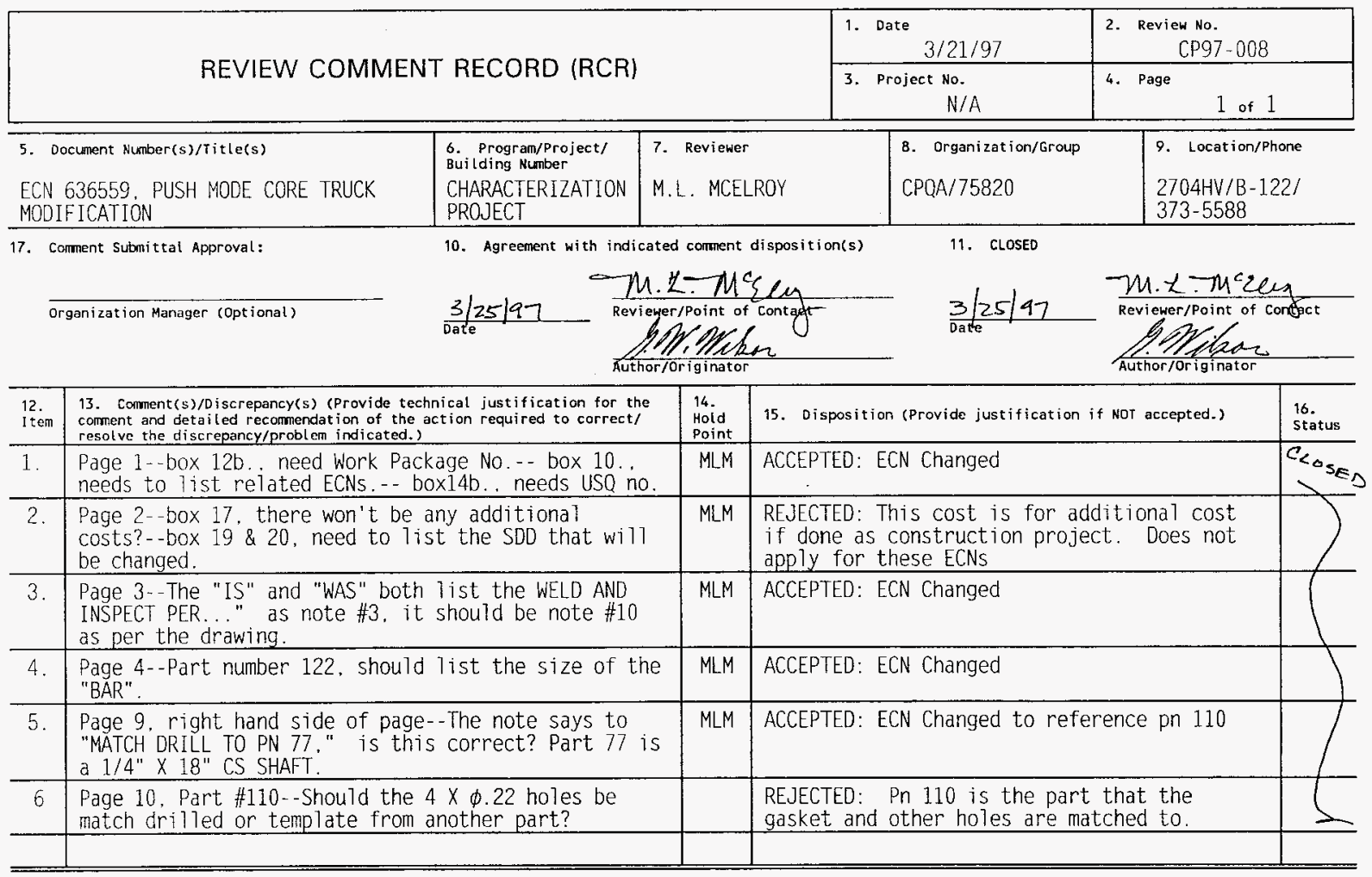




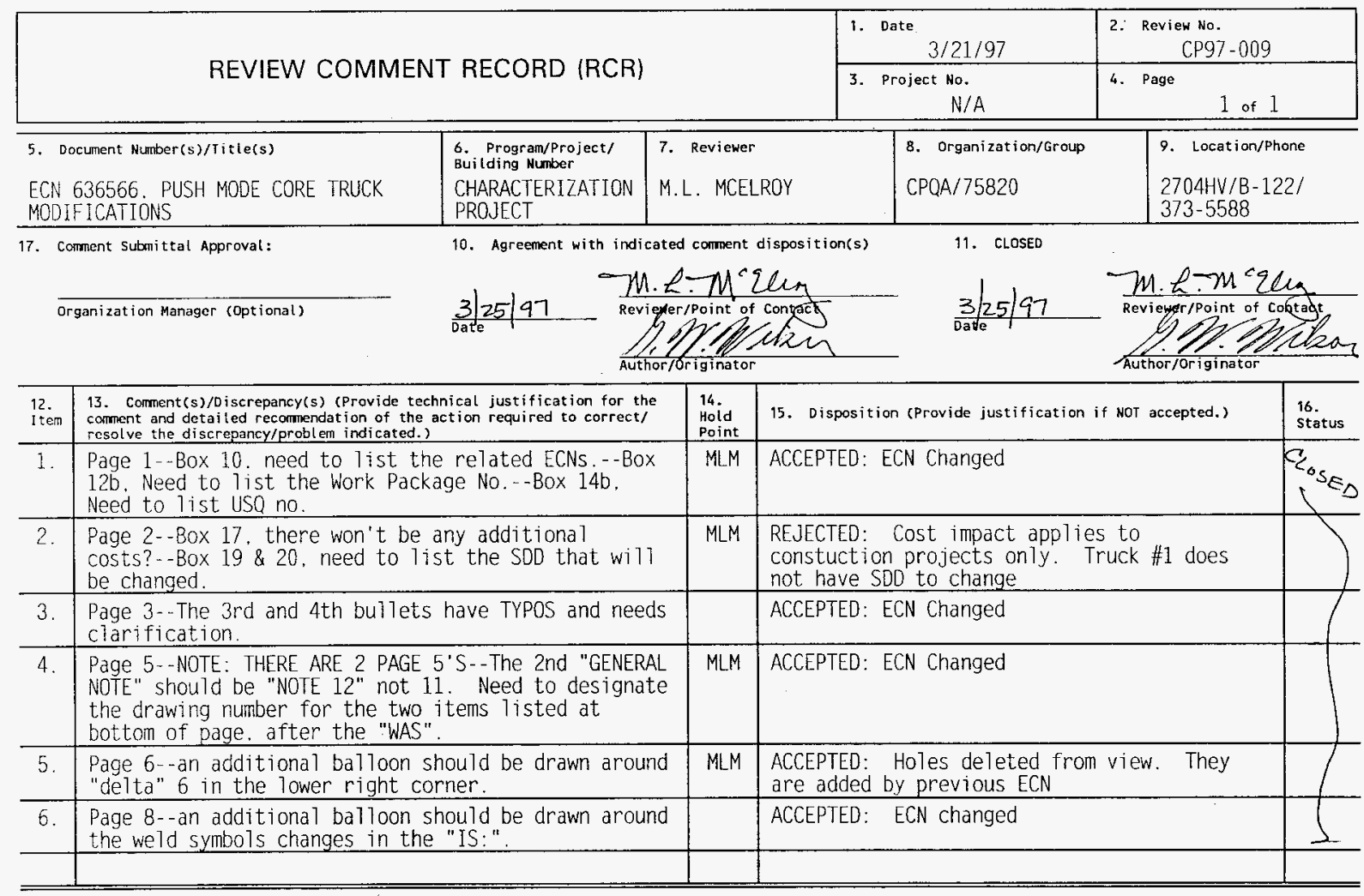




\begin{tabular}{|c|c|c|c|c|}
\hline & рә6ие4ว N3J :0 & $W 7 W$ & 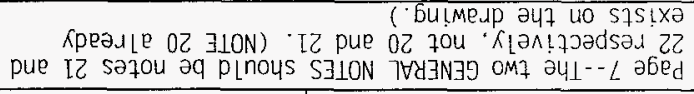 & 9 \\
\hline & 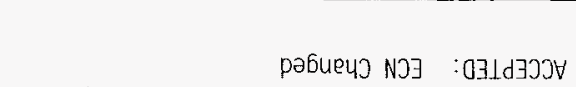 & $W 7 W$ & 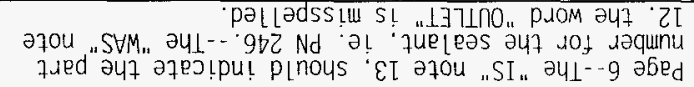 & G \\
\hline & 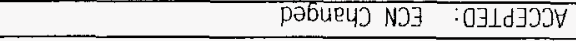 & WרW & 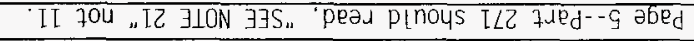 & b \\
\hline & ра6uе4ว $\mathrm{N} J \exists$ & W W & 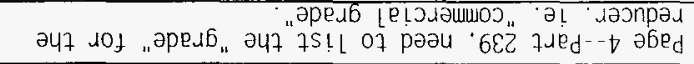 & $\varepsilon$ \\
\hline & 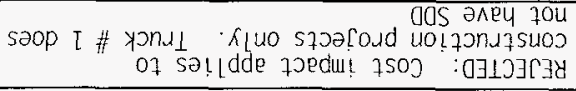 & Wาพ & 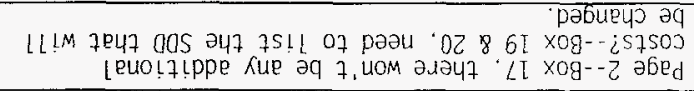 & Z \\
\hline 3 & рабиеบว NJJ & W7W & 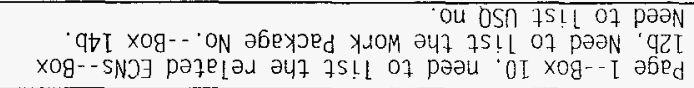 & I \\
\hline $\begin{array}{r}\text { snzezs } \\
.91\end{array}$ & 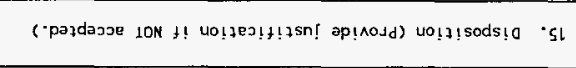 & $\begin{array}{l}\text { Fulod } \\
\text { Ploh } \\
\text { Pl }\end{array}$ & 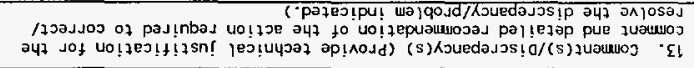 & $\begin{array}{l}\text { แəII I } \\
\cdot \mathrm{Cl}\end{array}$ \\
\hline
\end{tabular}

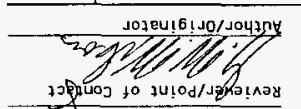

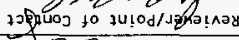
万b.twitim

$$
4 b / \text { szed }
$$

0350าง $\div$

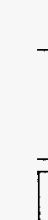

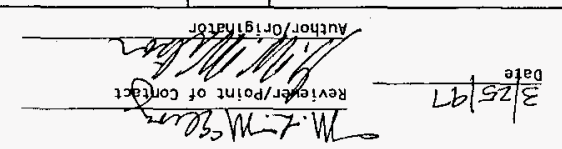

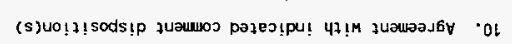

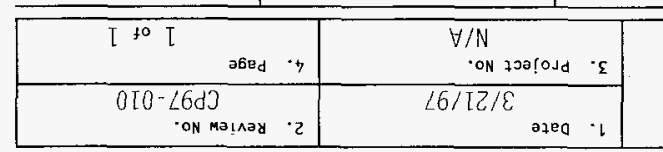

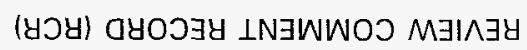




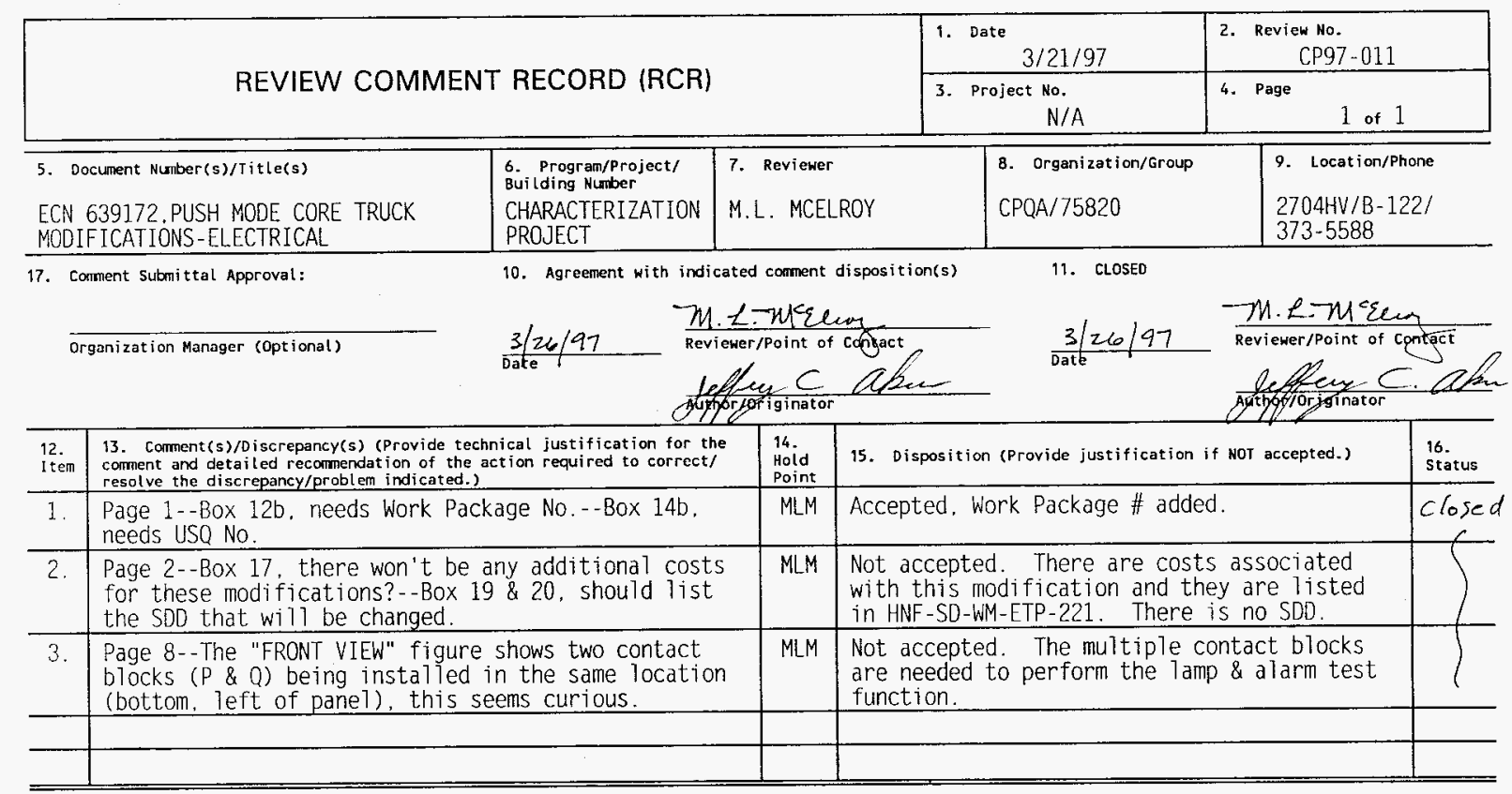




\begin{tabular}{|c|l|l|l|}
\hline \multirow{2}{*}{ REVIEW COMMENT RECORD (RCR) } & 1. Date & 2. Review No. & TF-97-0296 \\
\cline { 2 - 4 } & 3. Project No. & N4J2C & 4. Page \\
\hline
\end{tabular}

\begin{tabular}{l|l|l}
\hline 5. Document Number(s)/Title(s) & $\begin{array}{l}\text { 6. Program/Project/ } \\
\text { Building Number } \\
\text { Characterization } \\
\text { Project }\end{array}$ & $\begin{array}{l}\text { 7. Reviewer } \\
\text { Rick Freeman }\end{array}$ \\
\hline
\end{tabular}

17. Comment Subnittal Approval:

10. Agreement with indicatom coppent $\mathrm{g} /$ sposition(s)

\begin{tabular}{l|l}
\hline 8. organization/Group & 9. Location/Phone \\
Field Equipment & $2704 \mathrm{HV} / 373-1130$ \\
Engineering/8C 440 & \\
\hline
\end{tabular}

$\overline{\text { Organization Manager (Optional) }}$
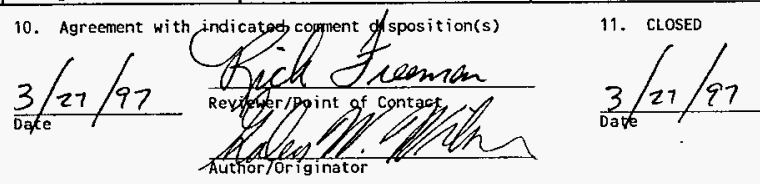

ก

\begin{tabular}{|c|c|c|c|c|}
\hline $\begin{array}{l}\text { 12. } \\
\text { Item }\end{array}$ & $\begin{array}{l}\text { 13. Comment }(s) / 0 \text { iscrepancy(s) (Provide technical justification for the } \\
\text { coment and detailed recomentat ion of the action required to correct/ } \\
\text { resolve the discrepancy/problem indicated.) }\end{array}$ & $\begin{array}{l}14 . \\
\text { Hoid } \\
\text { Point }\end{array}$ & 15. Disposition (Provide justification if NOT accepted.) & $\begin{array}{l}16 . \\
\text { Status } \\
\end{array}$ \\
\hline 1 & Page 1 - Block 3 , change the organization code. & & ACCEPTED: ECN changed. & CLOSED \\
\hline 2 & $\begin{array}{l}\text { Page } 1 \text { - Block 10. delete the N/A and add "See Block } \\
\text { 14b". }\end{array}$ & & ACCEPJED: ECN changed. & -6 \\
\hline 3 & Page 1 - Block 12b. . add work package number. & & ACCEPTED: ECN changed. & \\
\hline 4 & $\begin{array}{c}\text { Page } 1 \text { - Block 14a., add a check mark for "Criteria } \\
\text { Change". }\end{array}$ & & ACCEPTED: ECN changed. & \\
\hline 5 & $\begin{array}{l}\text { Page } 1 \text { - Block 14b.. correct spelling of } \\
\text { independent. Add "See page } 3 \text { for } \\
\text { additional justification and related ECN's. } \\
\text { This note should be consistent with all } \\
\text { three ECN's (636559, 636566. 636567). }\end{array}$ & & ACCEPTED: ECN changed. & $\downarrow$ \\
\hline & & & & \\
\hline & & & & \\
\hline & & & & \\
\hline & & & & \\
\hline & & & & \\
\hline
\end{tabular}




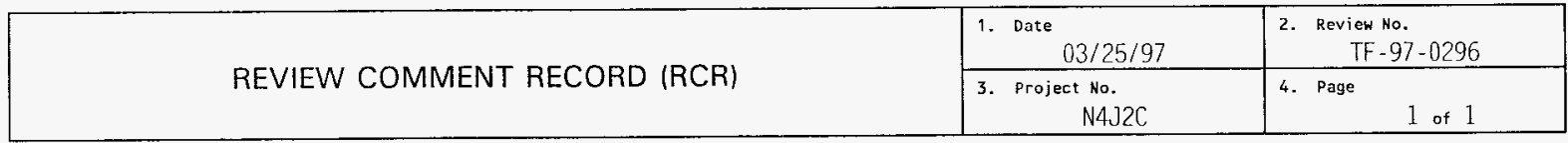

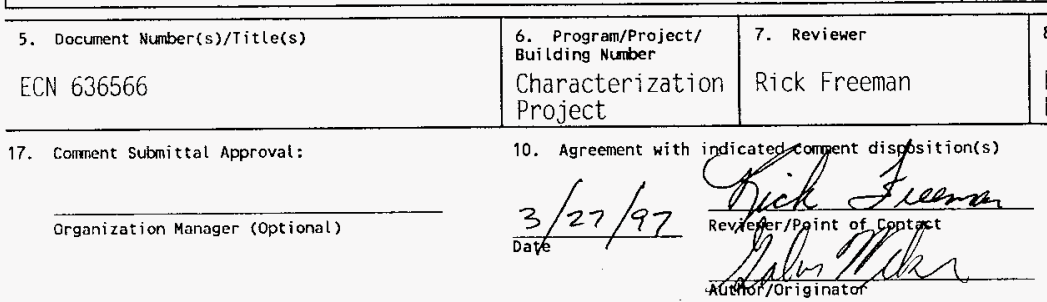

\begin{tabular}{|c|c|}
\hline 8. Organization/Group & 9. Location/Phone \\
\hline $\begin{array}{l}\text { Field Equipment } \\
\text { Engineering/8C } 440\end{array}$ & 2704HV/373-1130 \\
\hline
\end{tabular}

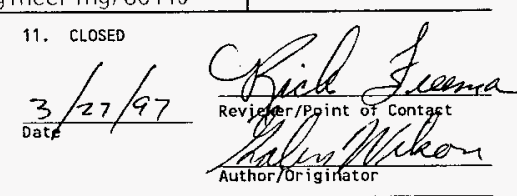

\begin{tabular}{|c|c|c|c|c|}
\hline $\begin{array}{l}12 . \\
\text { Itcm }\end{array}$ & $\begin{array}{l}\text { 13. Comment(s)/Discrepancy(s) (Provide technical justification for the } \\
\text { commintent and detaited recormendation of the action requi red to correct/ } \\
\text { resolve the discrepancy/problem indicated.) }\end{array}$ & $\begin{array}{l}14 . \\
\text { Hoid } \\
\text { Point }\end{array}$ & 15. Disposition (Provide justification if NOT accepted.) & $\begin{array}{l}16 . \\
\text { Status }\end{array}$ \\
\hline 1 & Page 1 - Block 10, add See Block 14b. & & ACCEPTED: ECN changed. & Cose \\
\hline 2 & Page 1 - Block $14 a$. add a check mark for "Criteria & & ACCEPTED: ECN changed. & - \\
\hline 3 & $\begin{aligned} \text { Page } 1 \text { - Block 14b, the note for see page } 3 \text { should } \\
\text { be consistent between all three ECN's } \\
(636559,636566,636567) .\end{aligned}$ & & ACCEPTED: ECN changed. & \\
\hline 4 & $\begin{array}{c}\text { Page } 4 \text { - Part no. 16, thread size shouid be } 1-12 \\
\text { vs. } 1-16 .\end{array}$ & & ACCEPTED: ECN changed. & \\
\hline 5 & $\begin{array}{c}\text { Page } 5 \text { - Two new note } 11 \text {. Align the notes. Two } \\
\text { page } 5 \text { 's. }\end{array}$ & & ACCEPTED: ECN changed. & \\
\hline 6 & $\begin{array}{l}\text { Page 6 - Lower right hand corner of the assembly, } \\
\text { should the revision } 6 \text { triangle be in the } \\
\text { revision cloud? }\end{array}$ & & ACCEPTED: ECN changed. & \\
\hline 7 & Page 11 - Add part no. 28 to assembly 18 . & & ACCEPTED: ECN changed. & \\
\hline 8 & $\begin{array}{l}\text { Page } 12 \text { - Discrepancy between thread type on part } \\
\text { no. } 25 \text { and part no. } 27 \text { (the } .637 \text { end) } \\
\text { UNC Vs. UNF. }\end{array}$ & & ACCEPTED: ECN changed. & 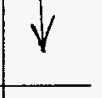 \\
\hline & & & & \\
\hline & & & & \\
\hline
\end{tabular}




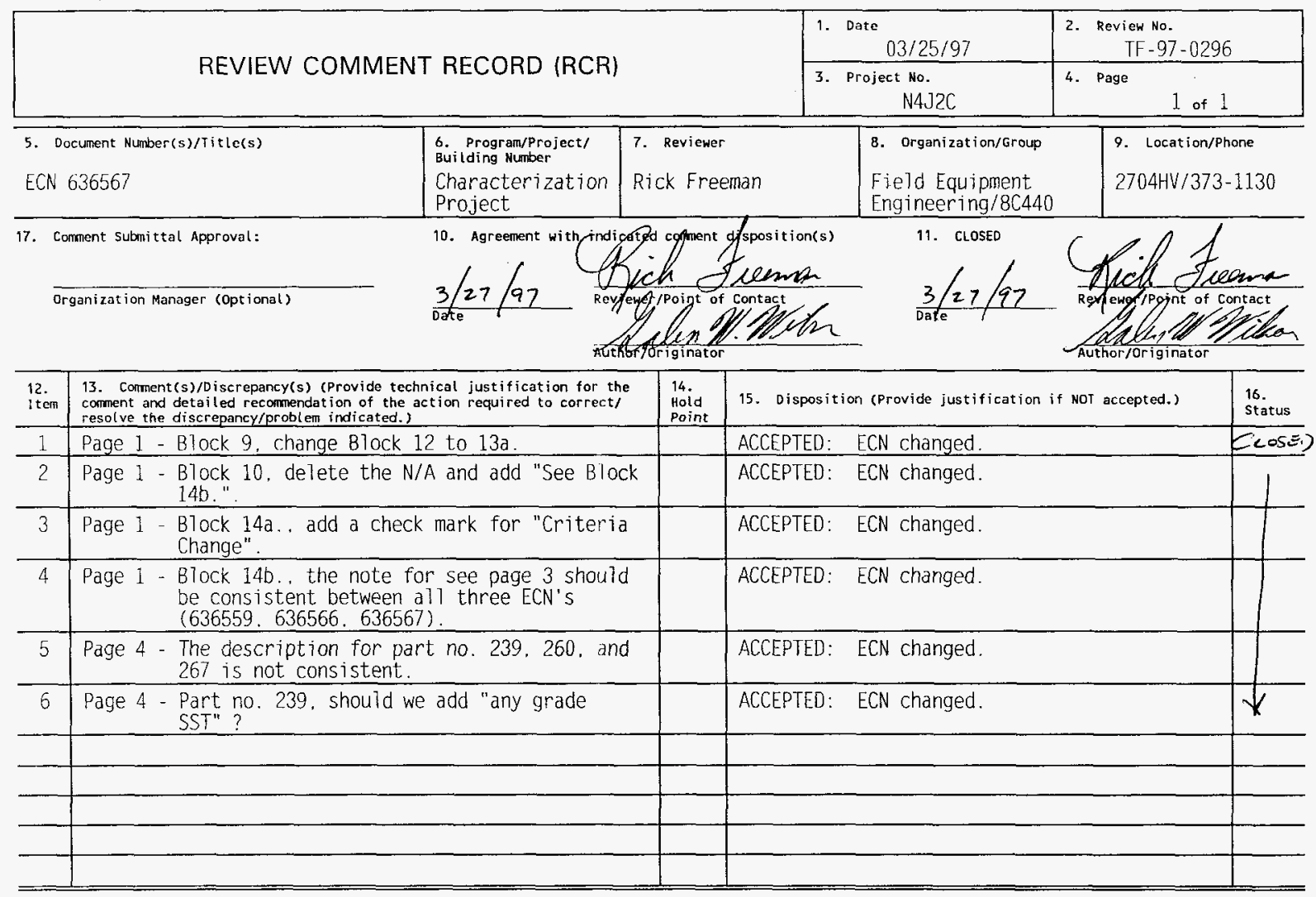




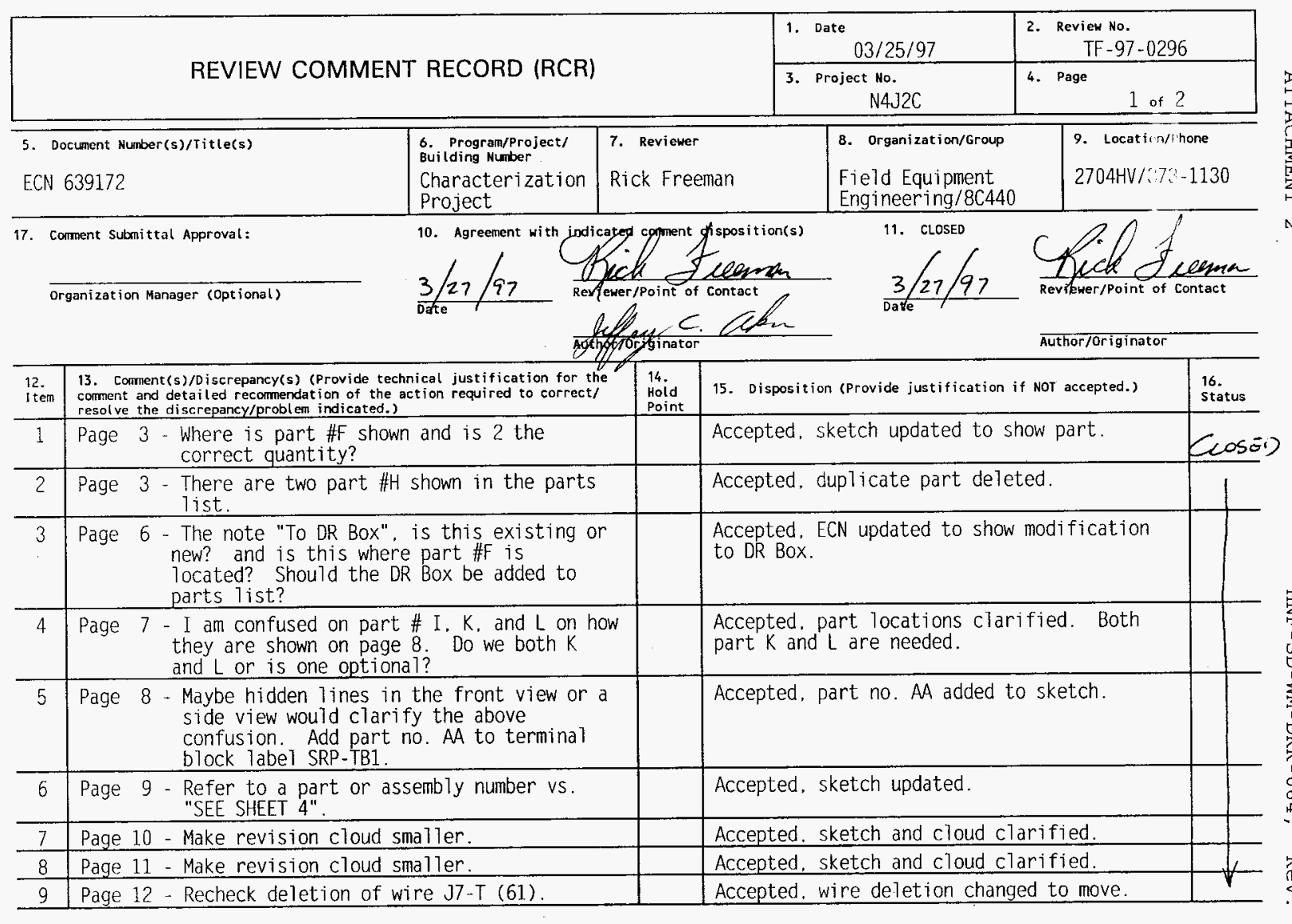

A-6400-090.1 (03/92) WEF011 


\begin{tabular}{|l|l|l|l|}
\hline \multirow{2}{*}{ REVIEW COMMENT RECORD (RCR) } & $\begin{array}{l}\text { 1. Date } \\
03 / 25 / 97\end{array}$ & $\begin{array}{c}\text { 2. Review No. } \\
\text { TF-97-0296 }\end{array}$ \\
\cline { 2 - 4 } & $\begin{array}{c}\text { 3. Project No. } \\
\text { N4J2C }\end{array}$ & $\begin{array}{c}4 . \text { Page } \\
2 \text { of } 2\end{array}$ \\
\hline
\end{tabular}

\begin{tabular}{|c|c|c|c|c|}
\hline $\begin{array}{l}12 . \\
\text { Item }\end{array}$ & $\begin{array}{l}\text { 13. Comment(s)/Discrepancy(s) (Provide technical justification for the } \\
\text { corment and detail ed recommendat ion of the action required to correct/ } \\
\text { resolve the discrepancy/problem indicated.) }\end{array}$ & $\begin{array}{l}14 . \\
\text { Hoid } \\
\text { Point }\end{array}$ & 15. Disposition (Provide justification if NOI accepted.) & $\begin{array}{l}16 . \\
\text { 5tatus }\end{array}$ \\
\hline 10 & $\begin{array}{l}\text { Page } 13 \text { - Both of the details on this page are } \\
\text { labeled "WAS". Also, make the revision } \\
\text { cloud smaller. }\end{array}$ & & Accepted, second WAS corrected to IS. & 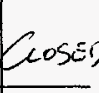 \\
\hline & & & & \\
\hline & & & & \\
\hline & & & & \\
\hline & & & & \\
\hline & & & & \\
\hline
\end{tabular}




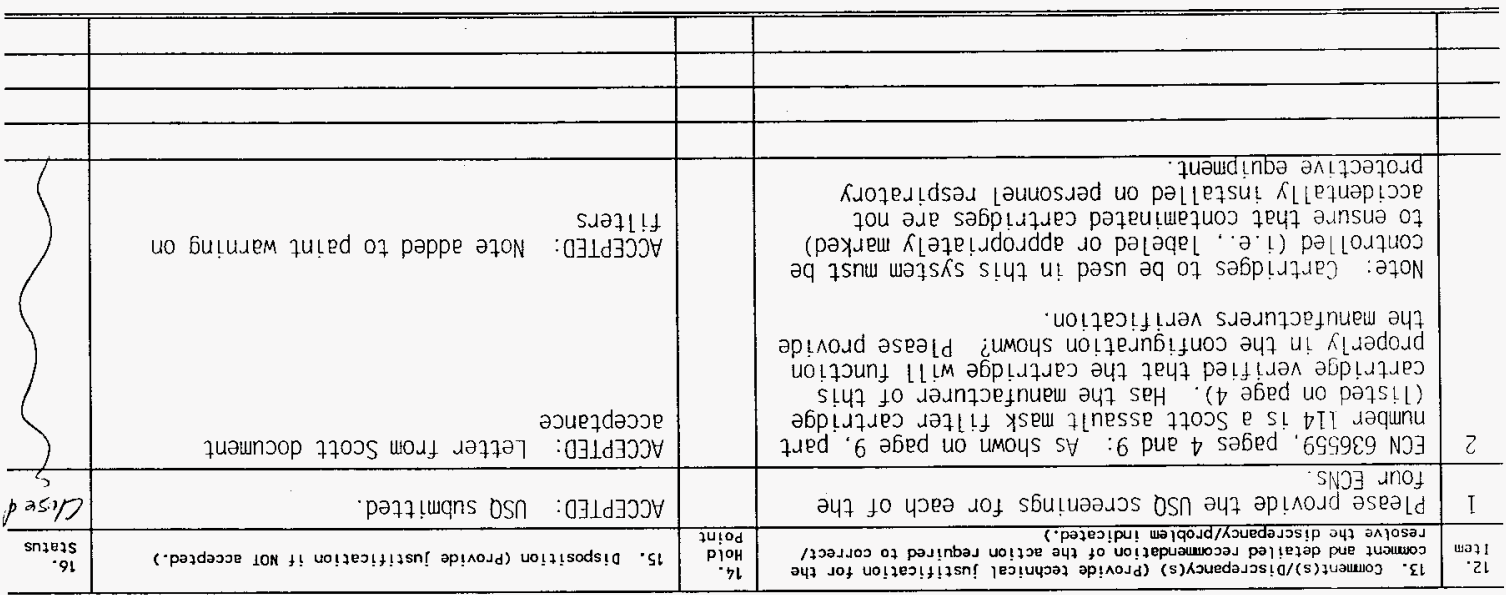
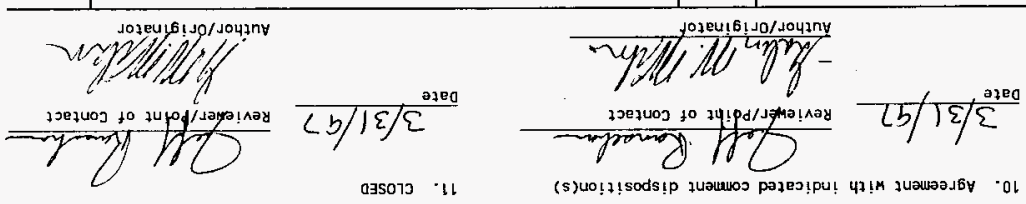

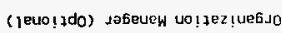

agso75 11

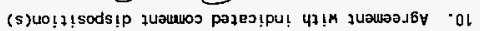

: jenodddy jejt!ulans quawoj - 2

\begin{tabular}{|c|c|c|c|c|}
\hline 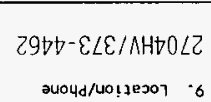 & $\begin{array}{r}\mathrm{SN} / \mathrm{Sd} \mathrm{J} \\
\text { dnosg/4o!zez!ue } 6 \text { so } \cdot 8\end{array}$ & 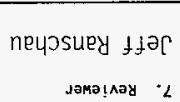 & 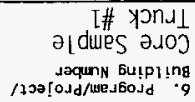 & 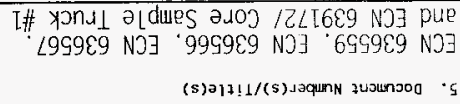 \\
\hline
\end{tabular}

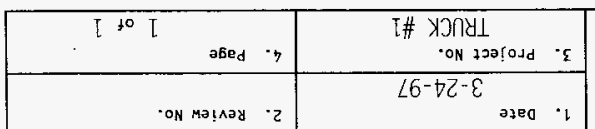

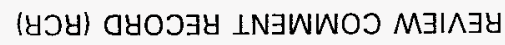




\begin{tabular}{|c|c|c|c|}
\hline \multirow{2}{*}{ REVIEW COMMENT RECORD (RCR) } & $\begin{array}{l}\text { 1. Date Review No. } \\
\text { GP3-970320 }\end{array}$ \\
\cline { 2 - 4 } & $\begin{array}{c}\text { 3. Project No. } \\
\text { CPO }\end{array}$ & $\begin{array}{c}\text { 4. Page } \\
1 \text { of } 2\end{array}$ \\
\hline
\end{tabular}

\begin{tabular}{|c|c|c|c|c|c|c|}
\hline \multicolumn{2}{|c|}{$\begin{array}{l}\text { 5. Document Number(s)/Title(s) } \\
\text { Insta17ation of Z-Purge on Truck \#1, } \\
\text { ECNs: } 636559.636566,636567,639172\end{array}$} & \begin{tabular}{|l|} 
6. Program/Project/ \\
Bui lding Number \\
CHARACTERIZATION \\
PROJECT \\
\end{tabular} & $\begin{array}{l}\text { 7. Reviewer } \\
\text { GP JANICEK }\end{array}$ & $\begin{array}{l}\text { 8. Organization/Group } \\
\text { TWRS DESIGN } \\
\text { AUTHORITY }\end{array}$ & \multicolumn{2}{|c|}{$\begin{array}{l}\text { 9. Location/Phone } \\
\text { 2704HV/A-104 } \\
376-2225\end{array}$} \\
\hline \multicolumn{2}{|c|}{ 17. Comment Submittal Approval: } & \multirow{2}{*}{\multicolumn{3}{|c|}{ 10. Agreement with indicated forment disposition(s) }} & \multirow{2}{*}{\multicolumn{2}{|c|}{$\begin{array}{l}\text { Reviewer/pbint of contact } \\
\text { Author/originator }\end{array}$}} \\
\hline \multicolumn{2}{|r|}{ Organization Manager (Optional) } & & & & & \\
\hline $\begin{array}{ll}12 . \\
\text { 1tem }\end{array}$ & $\begin{array}{l}\text { 13. Comment(s)/Discrepancy(s) (Provide tech } \\
\text { comment and detailed recormendation of the } \\
\text { resolve the discrepancy/problem indicated.) }\end{array}$ & $\begin{array}{l}\text { cal justification for th } \\
\text { ion required to correct/ }\end{array}$ & $\begin{array}{l}\text { Pit } \\
\text { Hoid } \\
\text { Point }\end{array}$ & \multicolumn{2}{|c|}{ i5. Disposition (Provide justification if NOT accepted.) } & $\begin{array}{l}16 . \\
\text { Status } \\
\end{array}$ \\
\hline 1. & A11 ECNs - Ensure block 12b is & illed in. & & \multicolumn{2}{|l|}{ ACCEPTED: Ecn revised } & $C \log C^{4}$ \\
\hline 2 & All ECNs - Ensure USQ is indica & ed in block $14 \mathrm{~b}$. & & \multicolumn{2}{|l|}{ ACCEPTED: ECn revised } & \\
\hline 3 & $\begin{array}{l}\text { A } 11 \text { ECNS - Need USQ completed ar } \\
\text { review prior to approval of the }\end{array}$ & $\begin{array}{l}\text { id submitted for } \\
\text { ECNs. }\end{array}$ & & \multicolumn{2}{|l|}{ USO completed. } & \\
\hline 4 & \multicolumn{2}{|c|}{$\begin{array}{l}\text { A11 ECNs - Include GP Janicek on distribution, block } \\
15 \text {. }\end{array}$} & & \multicolumn{2}{|l|}{ ACCEPTED: ECn revised } & \\
\hline 5 & \multicolumn{2}{|c|}{$\begin{array}{l}\text { A11 ECNs - Revise block } 21 \text { to indicate "Independent } \\
\text { Review". Need someone to signoff for ail } \\
\text { independent reviewers - probably Boger. }\end{array}$} & & \multicolumn{2}{|l|}{ ACCEPTED: ECn revised } & \\
\hline 6 & \multicolumn{2}{|c|}{$\begin{array}{l}\text { A11 ECNs - Revise block } 21 \text { to indicate proper } \\
\text { individuals required for approva?. }\end{array}$} & & \multicolumn{2}{|l|}{ ACCEPTED: Ecn revised } & \\
\hline 7 & \multicolumn{2}{|c|}{$\begin{array}{l}\text { All ECNs - In block } 21 \text { include MS Hendrix for } \\
\text { Environmental approval. }\end{array}$} & & \multicolumn{2}{|c|}{$\begin{array}{l}\text { ACCEPTED: Completed for ECNs } 636559 \text { and } \\
636567 \text {. Showed Michelle other two and she } \\
\text { said she didn't need to approve }\end{array}$} & \\
\hline 8 & \multicolumn{2}{|c|}{$\begin{array}{l}\text { General - On those ECNs where the Safety Equipment } \\
\text { List (SEL) is an impacted document, as indicated in } \\
\text { block 19. indicate which PNs the SEL has to address. }\end{array}$} & & \multicolumn{2}{|c|}{$\begin{array}{l}\text { ACCEPTED: Changed ECNS } 636657 \text { and } 639172 \text { to } \\
\text { include statement that pressure switches may } \\
\text { need to be added to SEL }\end{array}$} & \\
\hline
\end{tabular}




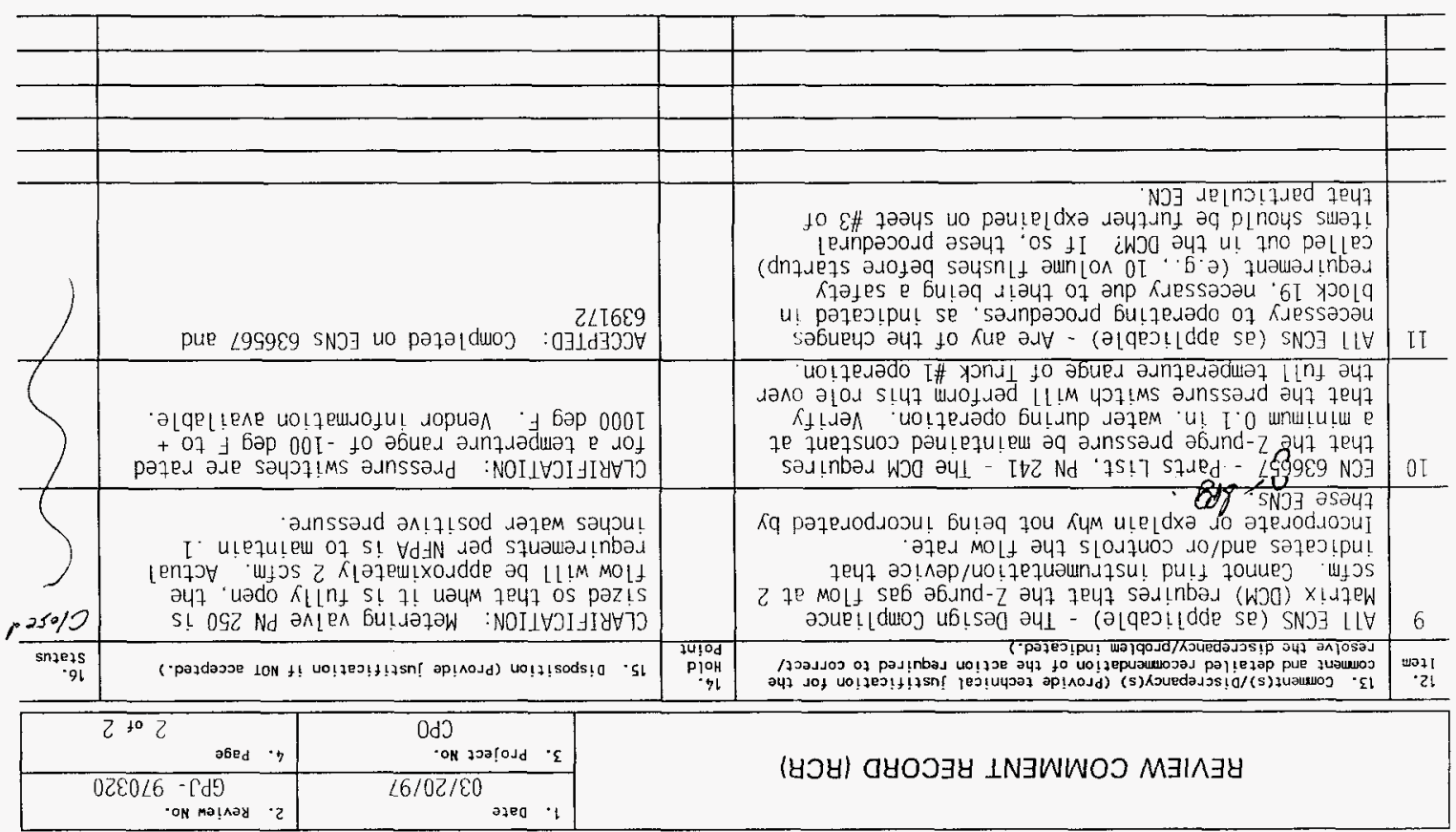




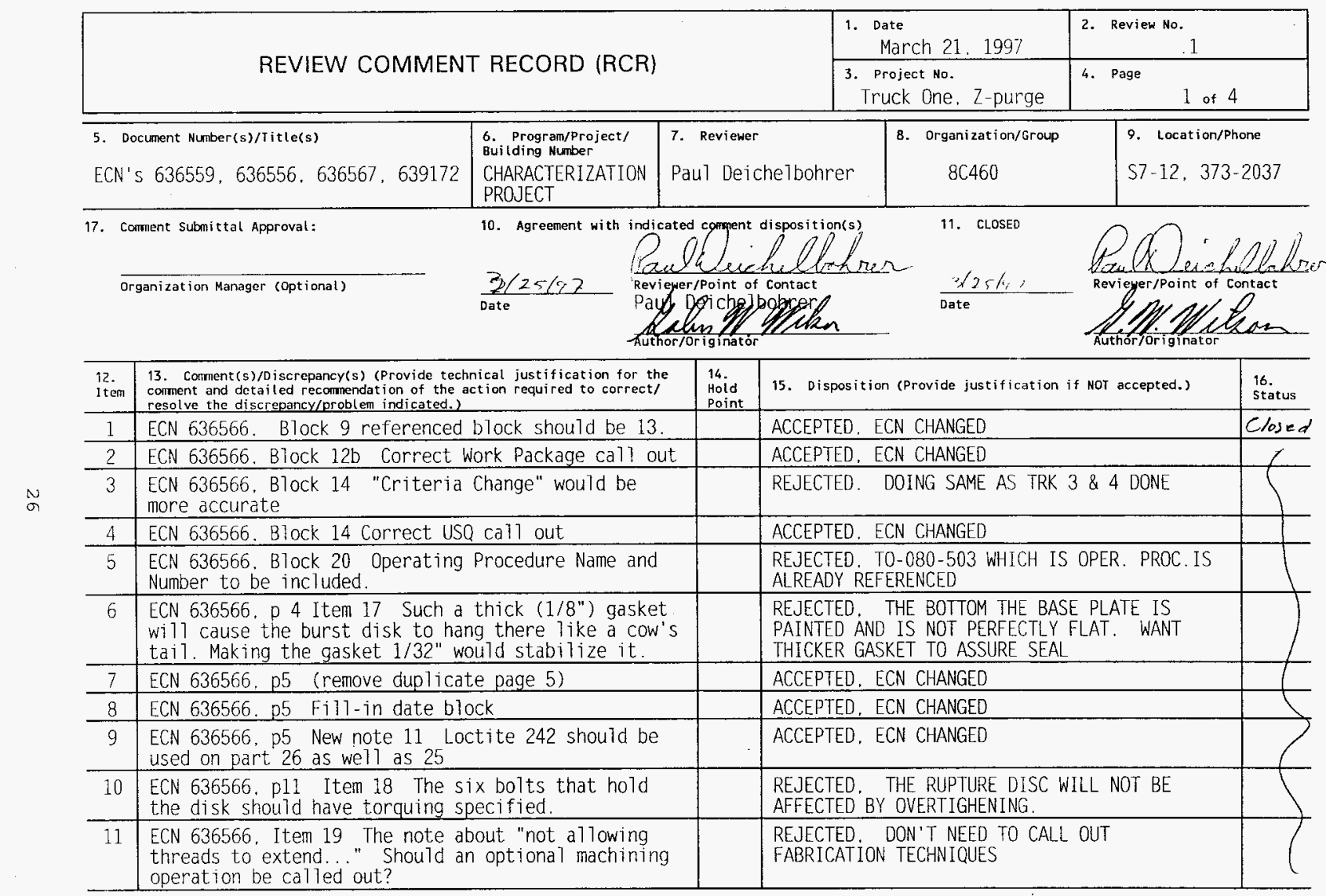




\begin{tabular}{|c|c|c|c|c|c|}
\hline & & & $\begin{array}{l}\text { 1. Date } \\
\text { March 21, } 1997\end{array}$ & 2. Review No. 1 & \\
\hline & REVIEW CUIVIVIEN) RECURU (RCR) & & $\begin{array}{l}\text { 3. Project Ho. } \\
\text { Truck One, Z-purge }\end{array}$ & 4. Page & \\
\hline $\begin{array}{l}12 . \\
1 \text { tem }\end{array}$ & $\begin{array}{l}\text { 13. Comment(s)/Discrepancy(s) (Provide technical justification for the } \\
\text { comment and detailed recommendation of the action required to correct/ } \\
\text { resolve the discrepancy/problem indicated.) }\end{array}$ & $\begin{array}{l}14 . \\
\text { Hoid } \\
\text { Point }\end{array}$ & 15. Disposition CProvide justificatior & f NOT accepted.) & $\begin{array}{l}\text { i6. } \\
\text { status }\end{array}$ \\
\hline 12 & $\begin{array}{l}\text { ECN } 636566 \text {. Drawing showing the assembly of Items } 26 \\
\text { and } 27 \text { should show the threads extending into item } \\
26 \text {. }\end{array}$ & & ACCEPTED. ECN CHANGED & & \\
\hline 13 & $\begin{array}{l}\text { ECN } 636566 \text {, p13 item } 24 \text { ID cal1 out should have a } \\
\text { decimal point instead of a comma. This is not a } \\
\text { metric dimension. }\end{array}$ & & ACCEPTED, ECN CHANGFD & & \\
\hline 14 & ECN 636559. B7ock 12 Need Work Package cal1 out & & ACCEPTED. ECN CHANGED & & \\
\hline 15 & ECN 636559 . BTock 14 INDEPENDENT NOT INDEPENDANT & & ACCEPTED, ECN CHANGED & & \\
\hline 16 & ECN 636559. Block 14 Need USQ ca11 out & & ACCEPTED. ECN CHANGED & & \\
\hline 17 & $\begin{array}{l}\text { ECN } 636559 \text { Block } 15 \text { Replace Smith with Drury Add } \\
\text { Freeman and McE Troy }\end{array}$ & & ACCEPTED, ECN CHANGED & & \\
\hline 18 & $\begin{array}{l}\text { ECN } 636559 \text {, p7 Detail } x \text { shows unistrut mounts both } \\
\text { on the same side of the horizontal pipe, but this } \\
\text { page show one on each side. Block } 20 \text { Include } \\
\text { Operating Procedure Ca } 71 \text { out }\end{array}$ & & ACCEPTED, ECN CHANGED & & \\
\hline 19 & $\begin{array}{l}\text { ECN } 636559 \text {, Reference to Note } 12 \text { for the unistrut } \\
\text { mounting doesn't seem to make sense. }\end{array}$ & & ACCEPTED. ECN CHANGED TO REF & RENCE NOTE 18 & \\
\hline 20 & $\begin{array}{l}\text { ECN } 636559 \text {, p9 Horizontal section of Item } 112 \\
\text { seems to be called out to be } 6 \text { feet long. Should be } \\
6 \text { inches. }\end{array}$ & & ACCEPTED, ECN CHANGED & & \\
\hline 21 & $\begin{array}{l}\text { ECN } 636559 \text {, Vertical lengths of Item } 112 \text { are not } \\
\text { specified. }\end{array}$ & . & $\begin{array}{l}\text { REJECTED, PART CALLED OUT AS } \\
\text { MEET DRAWING STANDARDS } \\
\end{array}$ & EUUIRED WHICH & \\
\hline 22 & $\begin{array}{l}\text { ECN } 636559 \text {. Match drilling note (center-right) } \\
\text { seems to have wrong PN's called out. PN } 14 \text { should } \\
\text { be } 12 \text { : PN } 77 \text { should be } 110 \text {. }\end{array}$ & & ACCEPTED, ECN CHANGED & & \\
\hline 23 & $\begin{array}{l}\text { ECN } 636559 \text {, pll Item } 111 \text { The ID of the gasket may } \\
\text { fit too tight on the pipe to be assembled. }\end{array}$ & & $\begin{array}{l}\text { REJECTED, WE WANT GASKET TO } \\
\text { OF PIPE. BELIEVE .060 INTER }\end{array}$ & $\begin{array}{l}\text { EAL AROUND OD } \\
\text { ERNCE WILL WORK }\end{array}$ & \\
\hline 24 & ECN 636559. PN 77 should be 110 . & & ACCEPTED, ECN CHANGED & & \\
\hline 25 & $\begin{array}{l}\text { ECN } 636559 \text {, Item } 113 \text { Pipe threads should be cut } \\
\text { after welding. }\end{array}$ & & $\begin{array}{l}\text { REJECTED, DRAWINGS TO NOT } \\
\text { FABRICATION TECHNIQUES }\end{array}$ & & \\
\hline
\end{tabular}




\section{REVIEW COMMENT RECORD (RCR)}

\begin{tabular}{|l|l|}
\hline $\begin{array}{l}\text { 1. Date } \\
\text { March 21.1997 }\end{array}$ & 2. Review No. \\
\hline $\begin{array}{l}\text { 3. Project No. } \\
\text { Truck One. Z-purge }\end{array}$ & 4. Page \\
\hline
\end{tabular}

\begin{tabular}{|c|c|c|c|c|}
\hline $\begin{array}{l}12 . \\
\text { Item }\end{array}$ & $\begin{array}{l}\text { 13. Coment(s)/Discrepancy(s) (Provide technical justification for the } \\
\text { coment and detailed recommendation of the action required to correct/ } \\
\text { resolve the discrepancy/problem indicated.) }\end{array}$ & $\begin{array}{l}14 . \\
\text { Hold } \\
\text { Point }\end{array}$ & 15. Disposition (Provide justification if NOT accepted.) & $\begin{array}{l}16 . \\
\text { Status }\end{array}$ \\
\hline 26 & ECN 636567. Total number of pages should be 14 & & ACCEPTED. ECN CHANGED & $\angle$ \\
\hline 27 & ECN 636567. BTock 9 Correct BLOCK cal1 out & & ACCEPTED, ECN CHANGED & \\
\hline 28 & ECN 636567. Block 12 Complete work package No. & & ACCEPTED. ECN CHANGED & \\
\hline 29 & ECN 636567. Block 14 Complete USQ cal1 out & & ACCEPTED. ECN CHANGED & \\
\hline 30 & ECN 636567, Block 15 Add Dale and Stubbs & & REJECTED, R. FREEMAN IS TRUCK \# 1 COG & \\
\hline 31 & $\begin{array}{l}\text { ECN } 636567 \text {, Block } 19 \text { Bungee cord and top vertical } \\
\text { sea? wi11 wear out. They should be checked in the } \\
\text { Periodic Maintenance Program. }\end{array}$ & & ACCEPTED, BUT DOES NOT REQUIRE ECN CHANGE & \\
\hline 32 & $\begin{array}{l}\text { ECN } 636567 \text {, p3 wording should reflect that ECN } \\
636566 \text { also installs the Burst Disk }\end{array}$ & & ACCEPTED, ECN CHANGED & \\
\hline 33 & ECN 636567. p4 Item 239 needs material call out & & ACCEPTED, ECN CHANGED & \\
\hline 34 & $\begin{array}{l}\text { ECN } 636567 \text {, Item } 252 \text { Dial Dia call out is wrong. } \\
\text { Probably should be } 21 / 2^{\prime \prime}\end{array}$ & & ACCEPTED, ECN CHANGED & \\
\hline 35 & $\begin{array}{l}\text { ECN 636567, p8 "Page } 8 \text { of } 13 \text { " should be "page } 8 \text { of } \\
14^{\prime \prime}\end{array}$ & & ACCEPTED, ECN CHANGED & \\
\hline 36 & $\begin{array}{l}\text { ECN } 636567 \text {, p10 Compared to page 13, the section } \\
\text { line BB-BB is either in wrong location or wrong } \\
\text { orientation. }\end{array}$ & & $\begin{array}{l}\text { ACCEPTED, ADDED NOTE TO VIEW STATING THAT } \\
\text { SAMPLER HOIST. WEATHER COVER, ETC NOT SHOWN } \\
\text { FOR CLARITY }\end{array}$ & \\
\hline 37 & $\begin{array}{l}\text { ECN } 636567 \text {, Bubbles for item } 244 \text { and } 247 \text { should be } \\
\text { included in drawing }\end{array}$ & & ACCEPTED, ECN CHANGED & \\
\hline 38 & $\begin{array}{l}\text { ECN } 636567 \text {, p11 Section } Z-Z \text { There should be a } \\
\text { note specifying inlet/outiet for filter. }\end{array}$ & & ACCEPTED. ADDED TO EXISTING NOTE & \\
\hline 39 & $\begin{array}{l}\text { ECN } 636567 \text { TYP MTG DETAIL wi11 welding to the } \\
\text { frame be aljowed at all locations that the tube is } \\
\text { to be mounted? }\end{array}$ & & ACCEPTED, ECN CHANGED & \\
\hline 40 & ECN 636567 . Bubble for item 247 should be included & & ACCEPTED. ECN CHANGED & \\
\hline 41 & ECN 636567. p12 Parts shouid be bubbled. & & ACCEPTED, ECN CHANGED & \\
\hline
\end{tabular}




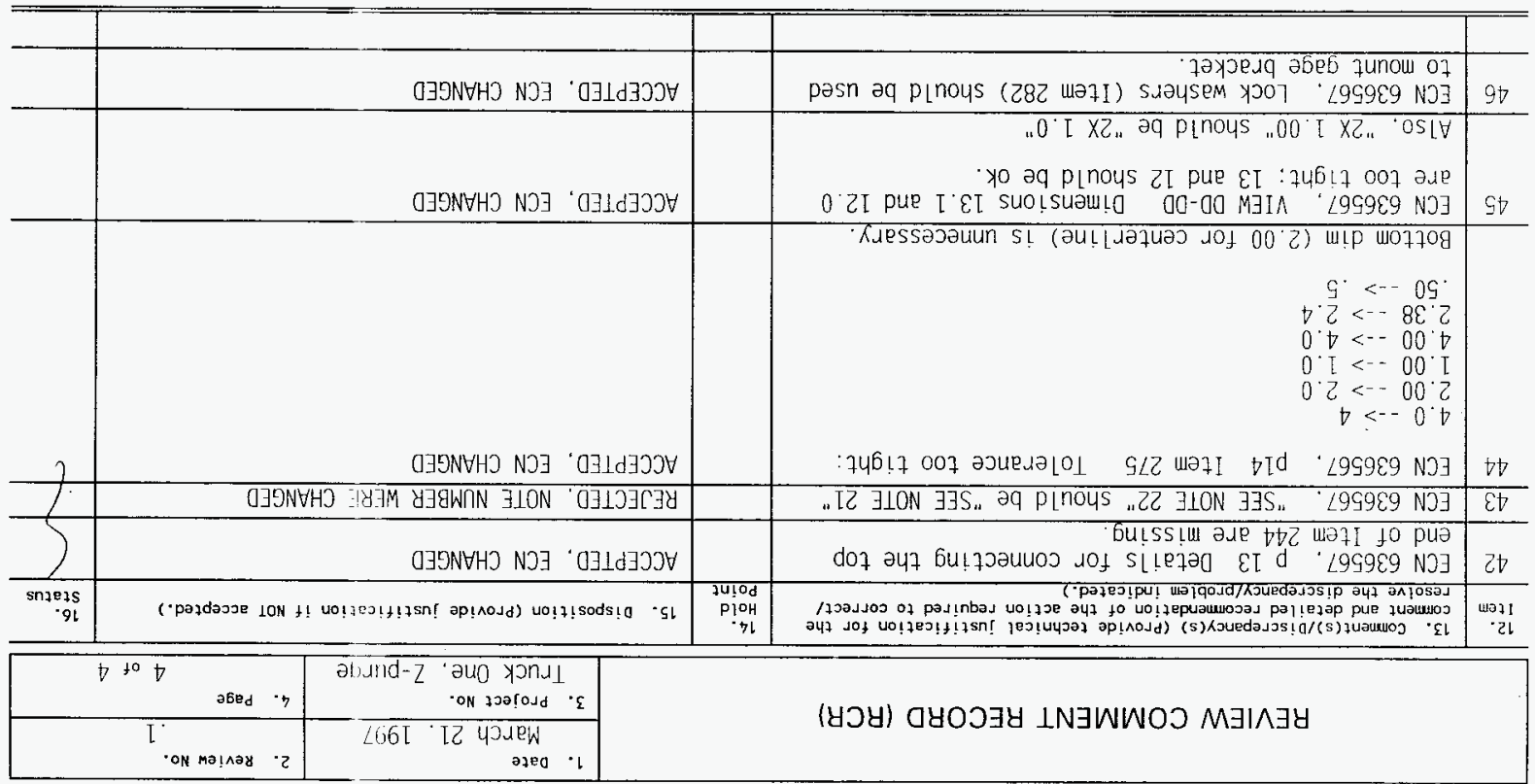




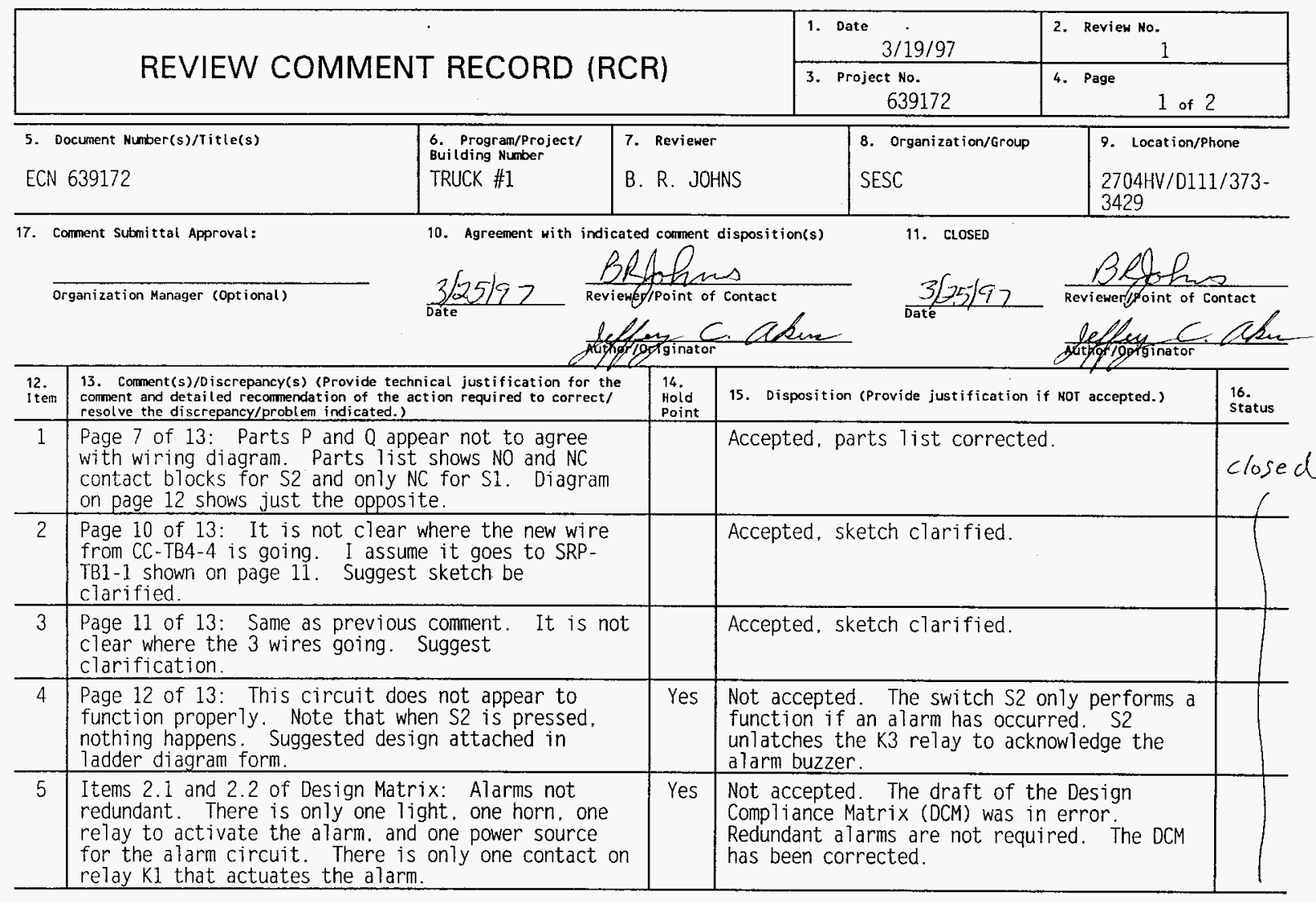




\section{REVIEW COMMENT RECORD (RCR)}

\begin{tabular}{|c|l|}
\hline 1. Date & \multicolumn{2}{|l|}{ 2.. Review No. } \\
$3 / 19 / 97$ & 4. Page \\
\hline $\begin{array}{c}\text { 3. Project No. } \\
639172\end{array}$ & \\
\hline
\end{tabular}

\begin{tabular}{|c|c|c|c|c|}
\hline $\begin{array}{l}12 . \\
\text { 1 tem }\end{array}$ & $\begin{array}{l}\text { 13. Corment(s)/Discrepancy(s) (Provide technical justification for the } \\
\text { comment and detailed recomnendation of the act ion required to correct/ } \\
\text { resolve the discrepancy/problem indicated.) }\end{array}$ & 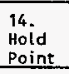 & 15. Disposition (Provide justification if NOI accepted.) & $\begin{array}{l}16 . \\
\text { Status }\end{array}$ \\
\hline 6 & $\begin{array}{l}\text { Item } 11 \text { of Design Matrix: Power failure to the } \\
\text { alarm circuit wi } 11 \text { cause no alarm. The current } \\
\text { design shows that if } \mathrm{k} 3 \text { relay fails, then there } \\
\text { would be no audible alarm. }\end{array}$ & Yes & $\begin{array}{l}\text { Not accepted. A complete power failure } \\
\text { would also remove power from the SR weather } \\
\text { cover equipment thereby eliminating any } \\
\text { electrically generated spark source. If } \\
\text { only the power to the alarm panel failed } \\
\text { then no lights would be on thus alerting the } \\
\text { operator to a potentially dangerous } \\
\text { condition. }\end{array}$ & Clo \\
\hline 8 & $\begin{array}{l}\text { Page } 7 \text { of } 13 \text { of ECN: I could not find in some } \\
\text { current catalogs, the part numbers for the Chicago } \\
\text { lamp body and lenses. Are these current numbers? }\end{array}$ & & $\begin{array}{l}\text { Not accepted. The part numbers listed are } \\
\text { current. However, for design reasons, the } \\
\text { parts in question were replaced. }\end{array}$ & \\
\hline 9 & $\begin{array}{l}\text { General: The alarm circuit appears to alarm when } \\
\text { the pressure is low and automatically resets when a } \\
\text { high pressure occurs. Is this acceptable to the } \\
\text { customer or should the alarm lock in and be manually } \\
\text { reset? I could not find design criteria defining } \\
\text { the aiarm condition needed. }\end{array}$ & & $\begin{array}{l}\text { Not accepted. The lights (green/red) will } \\
\text { indicate the status of the purge system } \\
\text { automatically. However, the audible alarm } \\
\text { does latch in and requires that the } \\
\text { acknowledge button (S2) be pressed to } \\
\text { silence it even if the alarm clears. This } \\
\text { function is not required. }\end{array}$ & \\
\hline & & & & \\
\hline & & & & \\
\hline & & & & \\
\hline & & & & \\
\hline & & & & \\
\hline & & & & \\
\hline
\end{tabular}


ATTACHMENT 2

DESIGN CALCULATIONNF-SD-WM-DRR-064, ReV. 0

(1) Drawing (2) Doc. No. EC 'N6.3\%172 (3) Page 1 of 2

(4) Building (5) Rev. $\bigcirc$ (6) Job No.

(7) Subject Alarm Circuit Indef Review)

(8) Originator Bruce $R$ Johns

(9) Checker

Date $3 / 19 / 97$

Date

(10) Alarm Circuit ladder diagram:

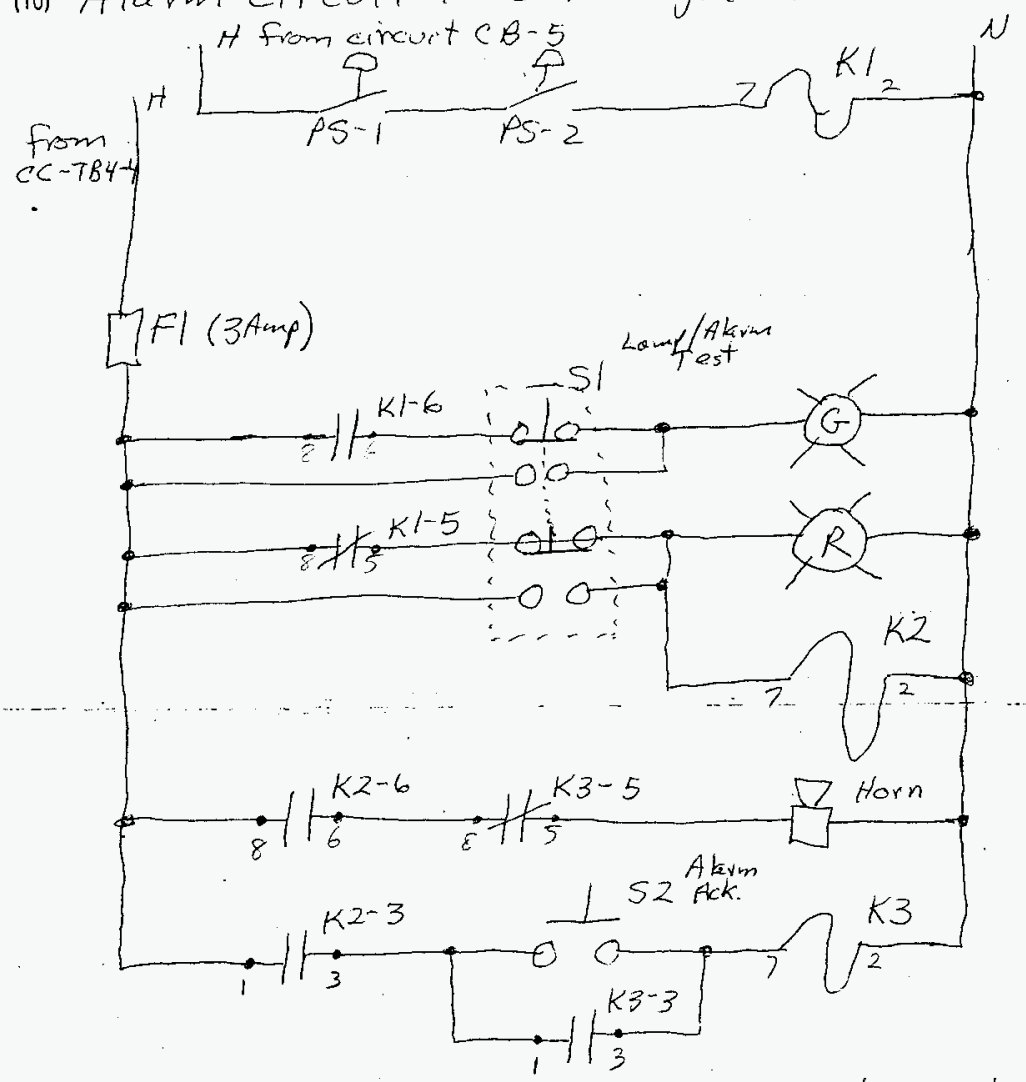

This will make existing circuit work. See explaimation on next page. However, I would not consider this a redundant circlet. BRA.

33 
HNF-SD-WM-DRR-064, REV 0

DESIGN CALCULATION JNNFSBEWMHAERRRE
Page A3-1

(1) Drawing (2) Doc. No (3) Page 2 of 2

(4) Building (5) Rev. (5) Job No

ATTACHMENT 3 Date

(9) Checker

Date

(10) Circuit function:

PS -1 and PS -2 normally closed and $K 1$ normally energized when pressure is high. K1-6 contact closes and green light is ON. Contact K/-5 is open and red light OFF and $k 2$ relay deenergized.

On low pressure or $k I$ relay power failure, $k 1$ deenergized. Contact kl-6 opens and green light is OFF, KI-5 contact closes and Red light $O N$ ard relay $K 2$ energizes. K2-6 contact closes and horn sounds. contact K2-3 closes and makes ready the acknowledge circuit.

Upon pressing 52 to acknowledge alarm, $k 3$ relay energizes and opens contact $k 3-5$ and terns horn OFF. contact $K 3-3$ closes and seals in $K 3$ relay to maintain acknowledge circuit upon pressure increase, $k 2$ relay deenergires and resets acknowledge circuit by resetting relay. $k 3$.

Bn g

34 
HNF-SD-WM-DRR-064, ReV. o

\section{FLAMMABLE GAS EQUIPMENT ADVISORY BOARD INTERPRETATION/RECOMMENDATION REPORT}

Board Members at Hearing:

J. T. Gregor (for R. A. Huckfeldt)

J. R. Kriskovich

C. M. Monasmith (for G. P. McDonald)
C. C. Scaief $I I I$

R. L. Schlosser

D. B. Smet
Report No:

FGEAB-97-032,

Rev. 1

Date: $4 / 8 / 97$

Requestor/Presenters: J.C. Akers, G.P. Janicek, J.S. Schofield, and G.W. Wilson.

Problems/Issue Statement:

Push Mode Core Sampling Truck 1 is currently operating under Non-Deminimus Temporary Exception Items 13 and 14 to the Ignition Source Control Requirements for the non-purged Shielded Receiver load cell and the Shielded Receiver weather cover, hoist, and limit switches in Facility Group 2 and 3 Waste Intruding Equipment. Design changes to provide Type $Z$ purge for the weather cover and provide direct venting to atmosphere for the Shielded Receiver have been developed to allow continued use of the equipment when Exception Items 13 and 14 expire. The FGEAB has been requested to evaluate the proposed changes for compliance with or equivalency to the Ignition Source Control requirements. Upon implementation of the Referenced ECNs, this Report supersedes the FGEAB-97-008 entries for the Shielded Receiver Load Cell and assembly for Truck 1 and the Shielded Receiver Weathercover - Hoist, Limit Switches for Truck 1. This revision incorporates Facility Group 1 applicability to the evaluation.

Equipment/Activity Description:

With the modifications proposed, the vent from the Shielded Receiver will be exhausted through a filtered outlet above and external to the Shielded Receiver weather cover and the Shielded Receiver weather cover will be equipped with Type $Z$ purge. The purge will be monitored by two externally mounted pressure switches located below the Shielded Receiver. A second filtered vent will be added to the bottom of the Shielded Receiver weather cover to ensure adequate purge flow. Audible and visual alarms will be provided for loss of purge pressure. Thus, the normally sparking Shielded Receiver load cell, hoist motor, and limit switches will be isolated from the Shielded Receiver by the inclusion of purging and relocation of the Shielded Receiver vent outside the Shielded Receiver weathercover. The externally mounted pressure switches (United Electric Controls Model JC120-521) are qualified to NFPA C1ass I, Divisions 1 \& 2 , Group $B$ requirements. All normally sparking components are isolated from the Waste Intruding Equipment (Shielded Receiver and drill string) by a single (non-hermetic) isolation barrier, making application of ISC 2 the appropriate requirement and providing Type $Z$ purge in compliance with ISC 2 requirements. 


\section{FLAMMABLE GAS EQUIPMENT ADVISORY BOARD INTERPRETATION/RECOMMENDATION REPORT}

\section{Equipment/Activity Description: (continued)}

The seal enclosure provided by the shielded receiver is evaluated to be an acceptable isolation barrier between the waste intruding equipment and the weathercover. This allows classification within the weather cover as equivalent to a Dome region. The pressure switches are located within the Ex-Tank Region due to their proximity to the Shielded Receiver weather cover vents.

References: ECNs 639172, 636559, 636566, \& 636567, Drawings H2-91653, Shts 1 \& 2

Applicable Authorization Basis: Standing Order 97-01, Rev. $0 \quad$ Dated: January 7, 1997

Does Equipment/Activity Comply with the AB Ignition Control Requirements? [X]Yes [ ]No [ ]0ther

If No, Does Equipment/Activity Provide Equivalent Design/Measures/Controls? N/A [ ]Yes [ ]No [ ]Other

Explanation/Justification: N/A

If Not Equivalent, What Changes are Necessary to Comply or Provide Equivalency? N/A

\section{FGEAB INTERPRETATION/RECONMENDATION:}

\begin{tabular}{|c|c|c|c|c|c|}
\hline $\begin{array}{c}\text { Equipment, Materials } \\
\& \text { Work Practices } \\
\text { (1) }\end{array}$ & $\begin{array}{l}\text { Location } \\
\text { (2) }\end{array}$ & $\begin{array}{l}\text { Ignition } \\
\text { Control } \\
\text { Set } \\
\text { (3) }\end{array}$ & $\begin{array}{l}\text { IC Set } \\
\text { Met } \\
\text { (4) }\end{array}$ & $\begin{array}{l}\text { FGEAB safety } \\
\text { Equivalence } \\
\text { Ruling } \\
\text { (5) }\end{array}$ & $\begin{array}{c}\text { Approved } \\
\text { Exception } \\
\text { (6) }\end{array}$ \\
\hline $\begin{array}{l}\text { Truck \#1: Shielded } \\
\text { Receiver load cell }\end{array}$ & $\begin{array}{l}\text { Facility } \\
\text { Group } \\
1,2 \& 3, \\
\text { Dome } \\
\text { Region* }\end{array}$ & ISC2 & $\begin{array}{l}\text { Yes, } \\
\text { with } \\
\text { type } 2 \\
\text { purge }\end{array}$ & & \\
\hline
\end{tabular}




\section{FLAMMABLE GAS EQUIPMENT ADVISORY BOARD INTERPRETATION/RECOMMENDATION REPORT}

Date: April 8, 1997

\begin{tabular}{|c|c|c|c|c|c|}
\hline $\begin{array}{c}\text { Equipment, Materials } \\
\text { \& Work Practices } \\
\text { (1) }\end{array}$ & $\begin{array}{l}\text { Location } \\
\text { (2) }\end{array}$ & $\begin{array}{c}\text { Ignition } \\
\text { Control } \\
\text { Set } \\
(3) \\
\end{array}$ & $\begin{array}{c}\text { IC Set } \\
\text { Met } \\
(4) \\
\end{array}$ & $\begin{array}{l}\text { FGEAB Safety } \\
\text { Equivalence } \\
\text { Ruling } \\
\text { (5) } \\
\end{array}$ & $\begin{array}{c}\text { Approved } \\
\text { Exception } \\
(6) \\
\end{array}$ \\
\hline $\begin{array}{l}\text { Truck \#1: Shielded } \\
\text { Receiver Weather } \\
\text { Cover Type Z Purge } \\
\text { Pressure Switches }\end{array}$ & $\begin{array}{l}\text { Facility } \\
\text { Group 1, } \\
\text { Ex-Tank } \\
\text { region; } \\
\text { Facility } \\
\text { Group 2, } \\
\text { Ex-Tank } \\
\text { region, } \\
\text { during } \\
\text { locally } \\
\text { waste } \\
\text { disturbing } \\
\text { activities }\end{array}$ & ISC2 & Yes & & \\
\hline $\begin{array}{l}\text { Truck \#1: Shielded } \\
\text { Receiver Weather } \\
\text { Cover Containing } \\
\text { Hoist and Limit } \\
\text { Switches }\end{array}$ & $\begin{array}{l}\text { Facility } \\
\text { Group } \\
1,2 \& 3 \\
\text { Dome } \\
\text { region* }\end{array}$ & ISC2 & $\begin{array}{l}\text { Yes, } \\
\text { with } \\
\text { type } z \\
\text { purge }\end{array}$ & & \\
\hline \multicolumn{6}{|c|}{$\begin{array}{l}\text { * Addition of Shielded Receiver venting outside the weather cover provides a seal } \\
\text { barrier between the Waste Intruding Equipment and the region housed within the Shielded } \\
\text { Receiver weathercover. Since the hoist shaft penetrates the Shielded Receiver, the } \\
\text { volume within the weather cover is treated as equivalent to a Dome Region. }\end{array}$} \\
\hline \multicolumn{6}{|c|}{ General Application of Finding, If Any: N/A } \\
\hline \multirow{2}{*}{$\begin{array}{l}\text { FGEAB Representative } \\
\text { Reviewer Signature: }\end{array}$} & \multirow{2}{*}{\multicolumn{3}{|c|}{$\begin{array}{l}\text { R. L. Schlosser (Signature on file) } \\
\text { D. B. Smet (Signature on file) }\end{array}$}} & Date: & $4 / 8 / 97$ \\
\hline & & & & Date: & $4 / 8 / 97$ \\
\hline
\end{tabular}




$$
\text { HNF-SD-WM-DRR-064, Rev. } 0
$$

ATTACHMENT 4 - Final Meeting Minutes

MEETING MINUTES

SUBJECT: Final Design Review Briefing, PMST Modification for Standing Order Compliance

\begin{tabular}{|c|c|c|c|c|}
\hline \multicolumn{2}{|l|}{$\begin{array}{l}\text { T0: } \\
\text { Distribution }\end{array}$} & \multicolumn{3}{|c|}{$\begin{array}{l}\text { BUILDING } \\
2704-\text { HV }\end{array}$} \\
\hline \multicolumn{2}{|l|}{$\begin{array}{l}\text { FROM: } \\
\text { GW Wilson }\end{array}$} & \multicolumn{3}{|c|}{$\begin{array}{l}\text { CHAIRMAN } \\
\text { GW Wi ISOn }\end{array}$} \\
\hline $\begin{array}{l}\text { DEPARTMENT-OPERATION-COMPONENT } \\
\text { Character ization Equipment Engineering }\end{array}$ & $\begin{array}{l}\text { AREA } \\
200-E\end{array}$ & $\begin{array}{l}\text { SHIFT } \\
\text { Day }\end{array}$ & $\begin{array}{l}\text { DATE OF MEETING } \\
3 / 26 / 97\end{array}$ & $\begin{array}{l}\text { NUMBER ATTENDING } \\
\quad 7\end{array}$ \\
\hline
\end{tabular}

A list of attendees is contained on the attached meeting roster. The meeting opened with a discussion of objectives. The next item of discussion concerned the possible revision of the Safety Equipment List and safety classification of components. It was agreed that the SEL would be addressed by final sign-off of $A B U$.

Revised copies of ECNs $636559,636566,636567$, and 639172 were passed out along with a summary of changes that had been completed on ECNs since previous review.

The changes to the ECNs were reviewed item by item against RCR comments to ensure that all reviewers were aware of all changes. Two minor errors were noted and corrected.

The design review closed without further discussion. 


\section{CST $\% 1$ Design HNF-SD-WM-DRR-064, Rev. 0}

MEETING REPORT (Continuation Sheet)

org.

Org. Code

Manager's Name

Meeting Location

Meeting Date

Meeting Time

2704HV/G108A

UPON COMPLETION OF THIS MEETING, HAVE ATTENDEES SIGN FORM.

Print Nane (Last, First, MI)

ALERS, TEFFERY Wilson, Galen

MSElroy, Michael L. Danickx, Gcunce-P BOGER, RM. Ranschan, IA. Pue $D$-ichelsotiRER
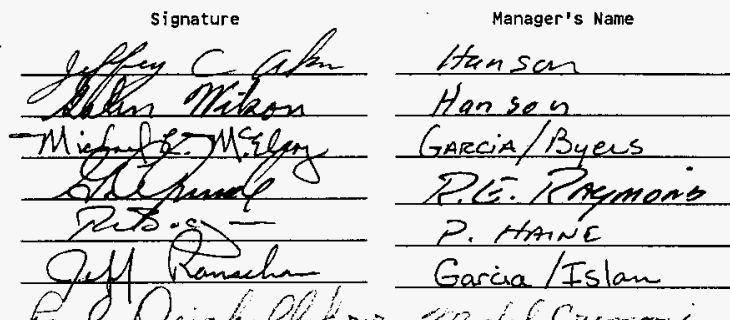

org. Code $Q 8 E 00$ $08 E 00$ 75820

$8 C 610$ 75830 80460 


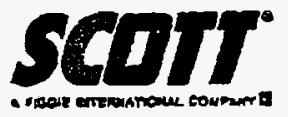

April 1. 1997

TO WHOM IT MAY CONCERN:

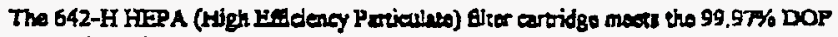
sendard at 16 or 32 Lmiraste gow with the tir flow goigg in either direction.

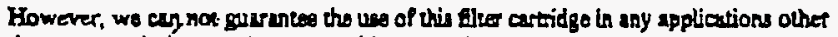
then as a respirator certridfo usad with nur repirstor fleppiece

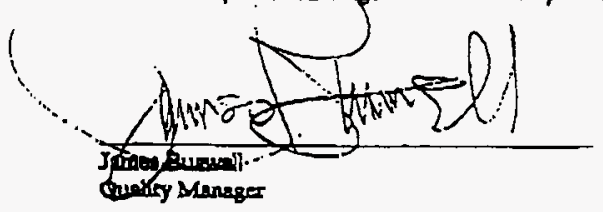

\begin{tabular}{|c|c|}
\hline Post-it" F & 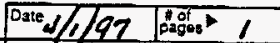 \\
\hline${ }^{\text {To }}$ Gouse ULSOD & From Giler Beown \\
\hline CO-Dopl RUOL - DNS. NC & Co. $500 \pi$ AvisT10N \\
\hline Phone" & Phone " $(800) 2 N 7-7257$ \\
\hline $373-423$ & 94) $291 \cdot 7746$ \\
\hline
\end{tabular}

